\title{
Feedback Linearisation for Nonlinear Vibration Problems
}

\author{
S. Jiffri, ${ }^{1}$ P. Paoletti, ${ }^{1}$ J. E. Cooper, ${ }^{2}$ and J. E. Mottershead ${ }^{1}$ \\ ${ }^{1}$ Centre for Engineering Dynamics, School of Engineering, The University of Liverpool, Brownlow Hill, Liverpool L69 3GH, UK \\ ${ }^{2}$ Department of Aerospace Engineering, University of Bristol, Queens Building, University Walk, Bristol BS8 1TR, UK \\ Correspondence should be addressed to S. Jiffri; s.jiffri@liv.ac.uk
}

Received 4 July 2013; Accepted 7 January 2014; Published 5 June 2014

Academic Editor: Nuno Maia

Copyright (C) 2014 S. Jiffri et al. This is an open access article distributed under the Creative Commons Attribution License, which permits unrestricted use, distribution, and reproduction in any medium, provided the original work is properly cited.

Feedback linearisation is a well-known technique in the controls community but has not been widely taken up in the vibrations community. It has the advantage of linearising nonlinear system models, thereby enabling the avoidance of the complicated mathematics associated with nonlinear problems. A particular and common class of problems is considered, where the nonlinearity is present in a system parameter and a formulation in terms of the usual second-order matrix differential equation is presented. The classical texts all cast the feedback linearisation problem in first-order form, requiring repeated differentiation of the output, usually presented in the Lie algebra notation. This becomes unnecessary when using second-order matrix equations of the problem class considered herein. Analysis is presented for the general multidegree of freedom system for those cases when a full set of sensors and actuators is available at every degree of freedom and when the number of sensors and actuators is fewer than the number of degrees of freedom. Adaptive feedback linearisation is used to address the problem of nonlinearity that is not known precisely. The theory is illustrated by means of a three-degree-of-freedom nonlinear aeroelastic model, with results demonstrating the effectiveness of the method in suppressing flutter.

\section{Introduction}

The effects of nonlinearity are everywhere to be seen in nature and it is true to say that many problems in structural dynamics are nonlinear, but in the past it has been convenient and easier to assume linearity. This linear analysis approach has necessarily led to conservative design of engineering structures that operate dynamically. In the last few decades the need to assume linearity has been increasingly challenged, mainly because of the high cost of fuel and materials and the need to preserve the earth's valuable natural resources. This is particularly true of the aerospace industries where the need to produce lightweight, fuel efficient aircraft is an unremitting pressure on design engineers. One problem is, of course, that nonlinear dynamic analysis is complicated and the useful linear-analysis methods, such as modal decomposition, are not applicable.

Inevitably active control will increasingly be seen as a solution for problems of nonlinearity in elastomechanics and aeroelasticity. One option is to design lightweight, fuel efficient aircraft and use active control to nullify the effects of the nonlinearity. By this approach, well-understood linear analysis methods can still be used. Feedback linearisation, described, for example, by Isidori [1], is a technique now well established in the active control community. Depending upon the implementation it is able to completely or partially linearise the nonlinear system. In the latter case there remains a (generally nonlinear) subsystem with dynamics that must be investigated to ensure stability. Of course, the linear part can be used for the attainment of a chosen control objective using conventional linear time invariant (LTI) techniques. An important aspect of feedback linearisation is that for the desired control objectives to be met precisely, the nonlinearity must be known in terms of its physical location, its type (e.g. cubic, quartic, free-play, etc.), and the numerical values of its parameters. This seems initially to pose a serious restriction on its application, but fortunately there exist techniques, such as adaptive feedback linearisation, that are able to account for incorrectly estimated nonlinear terms.

It is apparent from the literature that the feedback linearisation method, which holds much promise, has found only very limited application in active vibration suppression. 
Examples include Fossen and Paulsen [2] who applied adaptive feedback linearisation to the automatic steering of ships. In aeroelasticity, Ko and his colleagues [3-5] used feedback linearisation methods (including adaptation) and carried out a series of wind-tunnel tests on a two degree of freedom aerofoil with either one or two control surfaces. They found that with an erroneous nonlinear parameter, but without adaptation, their system reached a nonzero equilibrium, rather than the zero equilibrium that is usually sought. Monahemi and Krstic [6] employed adaptive feedback linearisation to update the aerodynamic parameters in their nonlinear model and thereby suppress wing-rock motion, a phenomenon triggered primarily by aerodynamic nonlinearities. Poursamad [7] implemented a hybrid neural-network controller for antilock braking with adaptive feedback linearisation to handle nonlinear and time-varying brake parameters. Bechlioulis and Rovithakis [8] developed a multiple-input, multiple-output tracking controller with adaptive feedback linearisation and Shojaei et al. [9] demonstrated the ability of adaptive feedback linearisation in aiding effective trajectory tracking in the presence of both parametric and nonparametric uncertainties in wheeled robots. Tuan et al. [10] designed a controller based on partial feedback linearisation of the nonlinear dynamics of a $3 \mathrm{D}$ overhead crane.

In this paper the problem of active control of nonlinear systems of the form

$$
\mathbf{A}_{1} \ddot{\mathbf{x}}+\mathbf{A}_{2} \dot{\mathbf{x}}+\mathbf{A}_{3} \mathbf{x}+\mathbf{f}_{\text {nl }}(\mathbf{x}, \dot{\mathbf{x}})=\mathbf{B u},
$$

representative of nonlinear vibration problems in elastomechanics and aeroelasticity, is considered. The vectors $\mathbf{x}, \dot{\mathbf{x}}$, and $\mathbf{u}$ typically contain the state variables and inputs, respectively. The nonlinearity $\mathbf{f}_{\mathrm{nl}}$ is given as a function of $\mathbf{x}$ and $\dot{\mathbf{x}}$ and the matrices $\mathbf{A}_{1}, \mathbf{A}_{2}, \mathbf{A}_{3}$, B represent the usual system parameters. This class of problem, characterised by the second-order matrix differential equation with additional nonlinearity confined to the left-hand side of (1), prevails to a very large extent in engineering mechanics and is therefore worthy of the special attention devoted to it in this paper. The classical output feedback linearisation [1] may be greatly simplified in the case of elastomechanical or aeroelastic systems described by (1). In particular,

(i) the essential theory is carried out entirely using the second-order matrix differential equation familiar to structural and aero-structural dynamicists, with the result that the need for repeated differentiation, usually described using the Lie-algebra notation, is rendered unnecessary,

(ii) a linear transformation applies between the state variable in (1) and the coordinates of the linearised system,

(iii) cancellation of the whole of the open-loop system dynamics (not just the nonlinear terms) results in a set of independent linear single degree of freedom systems for the application of conventional LTI control methods,

(iv) complete linearisation may be achieved with an equal number of actuators and sensors. If the number of actuators and sensors is less than the dimension of the system, then there will remain a nonlinear subsystem of dimension equal to the difference between the dimension of the full system and the number of sensors (and actuators). This subsystem can be made independent of the linearised part and methods are described to check its stability.

(v) Adaptive feedback linearisation is described for the treatment of an incorrect estimate of the nonlinearity. This makes use of Lyapunov stability criteria and results in a parameter update rule that evolves with time to ensure stability of the system.

The method is illustrated by means of a three degree of freedom aeroelastic system consisting of a flexible wing described in terms of two assumed modes in bending and torsion and a third degree of freedom that describes the angular motion of an underslung pylon-engine assembly. The parameters and dimensions of the system are carefully chosen to have realistic values.

\section{Active Feedback Linearisation}

Feedback linearisation [1] is a process whereby a nonlinear system is rendered linear by virtue of active control. Unlike Jacobian linearisation, it is exact, and does not entail an approximation at any stage. The method is implemented by transforming a nonlinear system into a linear one. For the class of second-order systems given by (1) considered in this paper,

$$
\begin{gathered}
\ddot{\mathbf{x}}=\mathbf{f}(\mathbf{x}, \dot{\mathbf{x}})+\mathbf{G u}, \quad \mathbf{f}(\mathbf{x}, \dot{\mathbf{x}})=\boldsymbol{\Psi} \mathbf{x}+\boldsymbol{\Phi} \dot{\mathbf{x}}+\boldsymbol{\Omega} \mathbf{f}_{\mathrm{nl}}, \\
\Psi=-\mathbf{A}_{1}^{-1} \mathbf{A}_{3}, \quad \boldsymbol{\Phi}=-\mathbf{A}_{1}^{-1} \mathbf{A}_{2}, \quad \boldsymbol{\Omega}=-\mathbf{A}_{1}^{-1}, \quad \mathbf{G}=\mathbf{A}_{1}^{-1} \mathbf{B},
\end{gathered}
$$

becomes

$$
\ddot{\mathbf{z}}=\bar{\Psi}_{\mathbf{z}}+\bar{\Phi} \dot{\mathbf{z}}+\overline{\mathbf{G}} \overline{\mathbf{u}}
$$

In these equations $\mathbf{u}$ and $\overline{\mathbf{u}}$ are, respectively, the actual (or physical) input applied to the nonlinear system and the socalled "artificial" input to the linear system. The matrices $\bar{\Psi}$, $\bar{\Phi}, \overline{\mathbf{G}}$ are dependent on the chosen inputs $\mathbf{u}$. The mapping from the nonlinear domain to the linearised domain is achieved through a nonsingular, linear, coordinate transformation. The actual input is designed to neutralise the effect of the nonlinearity, a procedure which can sometimes be achieved in full, and sometimes partially, as will be explained theoretically and by means of illustrative examples.

The process is quite straightforward for elastomechanical and aeroelastic systems described by second-order matrix differential equations with nonlinearity in the system parameters. The method described by Isidori [1] allows for greater generality, including nonlinearity in the input and output as well as in the system parameters, which is not required here and its omission leads to simplifications which aid the understanding of an otherwise fairly complicated procedure.

The feedback linearisation procedure classically, using the Lie algebra, entails repeated differentiation of each of the 
outputs with respect to time, until the input terms appear. The classical procedure is greatly simplified in the case of second-order matrix systems such as those in elastomechanics or aeroelasticity, as explained in the sequel. The present work addresses two cases. The first case is that of complete input-output linearisation, meaning a full complement of outputs and inputs at every degree of freedom of the system. In this case, since the number of outputs is equal to the dimension of the system, it is possible to linearise the entire nonlinear system. The complete dynamics of the original system are preserved during the transformation. In the second case, an incomplete system of equal numbers of inputs and outputs is assumed. In this case, as the number of inputs and outputs is less than the dimension of the overall model only a partial linearisation of the system can be achieved. The portion that remains untransformed will contribute to what is known as the internal dynamics, whose stability must be ensured for stability of the overall closed-loop system. This is achieved by examining the stability of the zero dynamics [1], which is obtained by setting all coordinates of the linearised subsystem to zero in the expressions for the internal dynamics. Expressions for the latter are obtained such that their time-derivatives are orthogonal to the inputs, rendering the zero dynamics uncontrollable. The zero dynamics may be either linear or nonlinear.

2.1. Complete Input-Output Linearisation (n-Inputs, $n$ Outputs). In the present case the number of inputs and outputs is equal to the dimension of the system. The matrices and vectors of the original nonlinear system, given in (2), have the dimensions $\Psi, \boldsymbol{\Phi}, \boldsymbol{\Omega}, \mathbf{G} \in \mathbb{R}^{n \times n} ; \mathbf{x}, \mathbf{u}, \mathbf{f} \in \mathbb{R}^{n \times 1}$ and those given by the desired linearised equation (3) by $\bar{\Psi}, \bar{\Phi}$, $\overline{\mathbf{G}} \in \mathbb{R}^{n \times n} ; \overline{\mathbf{z}}, \overline{\mathbf{u}} \in \mathbb{R}^{n \times 1}$. The first step is to choose the vector of actual inputs that cancels the nonlinearity,

$$
\mathbf{u}=\mathbf{G}^{-1}(\overline{\mathbf{u}}-\mathbf{f}(\mathbf{x}, \dot{\mathbf{x}})) .
$$

It can be seen that the nonlinearity is indeed cancelled by substituting (4) into (2). In fact, this is a special case where not only the nonlinearity, but also the complete open loop dynamics is cancelled by the choice of actual input. The result is the linearised system of independent, second-order, single degree of freedom equations

$$
\left(\begin{array}{c}
\ddot{x}_{1} \\
\ddot{x}_{2} \\
\vdots \\
\ddot{x}_{n}
\end{array}\right)=\left(\begin{array}{c}
\bar{u}_{1} \\
\bar{u}_{2} \\
\vdots \\
\bar{u}_{n}
\end{array}\right) .
$$

In fact, these equations are a special case of single degree of freedom equations, where each equation is simply a double integrator. Equation (5) is a particular form of (3), where also it is seen particularly that $\mathbf{x}=\mathbf{z}$. The simplicity of (5) is an advantage of the complete cancellation of the openloop dynamics in (4). The choice of the artificial input $\overline{\mathbf{u}}$ necessarily depends upon the control objective, for example, the assignment of a pair of complex conjugate poles in each of the systems in (5) to avoid resonances. Whatever the control objective is, it will result in the determination of gains defined here in terms of negative feedback as

$$
\begin{gathered}
\bar{u}_{1}=-\left[\begin{array}{ll}
\varsigma_{1} & v_{1}
\end{array}\right]\left\{\begin{array}{l}
x_{1} \\
\dot{x}_{1}
\end{array}\right\} \\
\bar{u}_{2}=-\left[\begin{array}{ll}
\varsigma_{2} & v_{2}
\end{array}\right]\left\{\begin{array}{l}
x_{2} \\
\dot{x}_{2}
\end{array}\right\} \\
\vdots \\
\bar{u}_{n}=-\left[\begin{array}{ll}
\varsigma_{n} & v_{n}
\end{array}\right]\left\{\begin{array}{l}
x_{n} \\
\dot{x}_{n}
\end{array}\right\} .
\end{gathered}
$$

In this special case where the entire open loop dynamics are cancelled, the control results in a closed-loop system that is comprised of $n$ decoupled, single degree of freedom subsystems. Then, having defined the artificial inputs, the actual inputs that provide the desired linearisation are determined from (4). It is seen that the nonlinearity in (2) is neutralised and the closed-loop system is indeed linear with the required dynamics.

If the actual input were chosen to cancel the nonlinearity alone (not the complete open-loop dynamics), then (4) would be replaced by

$$
\mathbf{u}=\mathbf{G}^{-1}\left(\overline{\mathbf{u}}-\mathbf{\Omega} \mathbf{f}_{\mathrm{nl}}\right),
$$

and the linearised equations would remain coupled (unlike (5)) and consequently the gains in (6) would take a different form, as

$$
\begin{aligned}
\left\{\begin{array}{c}
\bar{u}_{1} \\
\bar{u}_{2} \\
\vdots \\
\bar{u}_{n}
\end{array}\right\}= & -\left[\begin{array}{ccccccc}
\varsigma_{11} & v_{11} & \varsigma_{12} & v_{12} & \cdots & \varsigma_{1 n} & v_{1 n} \\
\varsigma_{21} & v_{21} & \varsigma_{22} & v_{22} & \cdots & \varsigma_{2 n} & v_{2 n} \\
\vdots & \vdots & \vdots & \vdots & \ddots & \vdots & \vdots \\
\varsigma_{n 1} & v_{n 1} & \varsigma_{n 2} & v_{n 2} & \cdots & \varsigma_{n n} & v_{n n}
\end{array}\right] \\
& \times\left\{\begin{array}{c}
x_{1} \\
\dot{x}_{1} \\
x_{2} \\
\dot{x}_{2} \\
\vdots \\
x_{n} \\
\dot{x}_{n}
\end{array}\right\} .
\end{aligned}
$$

Clearly, there are a greater number of control gains in (8) than in (6), which means that there is more control flexibility, which might be used, for example, to assign the eigenvectors as well as the eigenvalues. This may be readily achieved using methods such as that presented in [11] and is illustrated through a numerical example later on.

\subsection{Partial Input-Output Linearisation (m-Inputs, $m$-Outputs,} $m<n)$. The inputs and outputs (actuators and sensors), $\mathbf{u}, \mathbf{x}_{(1: m)} \in \mathbb{R}^{m \times 1}$, in equal numbers are now fewer than the dimension of the system. Linearisation results in similar 
expressions to those obtained for the complete input-output case presented above. Equation (2) is now rewritten as

$$
\begin{aligned}
\left\{\begin{array}{c}
\ddot{x}_{1} \\
\vdots \\
\ddot{x}_{m} \\
\ddot{x}_{m+1} \\
\vdots \\
\ddot{x}_{n}
\end{array}\right\}=\left\{\begin{array}{c}
f_{1}(\mathbf{x}, \dot{\mathbf{x}}) \\
\vdots \\
f_{m}(\mathbf{x}, \dot{\mathbf{x}}) \\
f_{m+1}(\mathbf{x}, \dot{\mathbf{x}}) \\
\vdots \\
f_{n}(\mathbf{x}, \dot{\mathbf{x}})
\end{array}\right\} \\
+\left[\begin{array}{ccc}
g_{1,1} & \cdots & g_{1, m} \\
\vdots & \ddots & \vdots \\
\frac{g_{m, 1}}{g_{m+1,1}} & \cdots & g_{m, m} \\
\vdots & \ddots & g_{m+1, m} \\
g_{n, 1} & \cdots & g_{n, m}
\end{array}\right]\left\{\begin{array}{c}
u_{1} \\
\vdots \\
u_{m}
\end{array}\right\},
\end{aligned}
$$

and the coordinate system which maps the original nonlinear system to the partially linearised system may be expressed as

$$
\left(\begin{array}{llll}
z_{1} & z_{2} & \cdots & z_{m}
\end{array}\right)^{T}=\left(\begin{array}{llll}
x_{1} & x_{2} & \cdots & x_{m}
\end{array}\right)^{T},
$$

which is identical to the full output feedback case, except of course that there are now only $m$ outputs. Further $(n-m)$ coordinates are needed and are chosen as coefficients of the orthonormal basis of the null space of $\mathbf{G}_{(1: n, 1: m)}^{T}$ so that

$$
\begin{gathered}
\left(\begin{array}{c}
x_{1} \\
\vdots \\
x_{n}
\end{array}\right)=\mathbf{V}\left(\begin{array}{c}
z_{m+1} \\
\vdots \\
z_{n}
\end{array}\right), \\
\mathbf{V}^{T} \mathbf{V}=\mathbf{I}_{(n-m) \times(n-m)}, \quad \mathbf{V}^{T} \mathbf{G}_{(1: n, 1: m)}=\mathbf{0}, \\
\mathbf{V} \in \mathbb{R}^{n \times(n-m)} .
\end{gathered}
$$

As with the full output feedback case, it is now necessary to choose actual inputs so that the nonlinearity is eliminated. This is achieved by

$$
\begin{gathered}
\mathbf{u}=\left[\mathbf{G}_{1: m, 1: m}\right]^{-1}\left(\overline{\mathbf{u}}-\mathbf{f}(\mathbf{x}, \dot{\mathbf{x}})_{(1: m, 1)}\right), \\
\mathbf{f}(\mathbf{x}, \dot{\mathbf{x}})_{(1: m, 1)}=\boldsymbol{\Psi}_{(1: m,:)} \mathbf{x}+\boldsymbol{\Phi}_{(1: m,:)} \dot{\mathbf{x}}+\boldsymbol{\Omega}_{(1: m,:)} \mathbf{f}_{\mathrm{nl}},
\end{gathered}
$$

and substitution of (12) into the upper partition of (9) leads to $m$ independent linear second-order systems with artificial inputs $\overline{\mathbf{u}}=\left\{\begin{array}{lll}\bar{u}_{1} & \cdots & \bar{u}_{m}\end{array}\right\}^{T}$, expressed as

$$
\left(\begin{array}{c}
\ddot{z}_{1} \\
\ddot{z}_{2} \\
\vdots \\
\ddot{z}_{m}
\end{array}\right)=\left(\begin{array}{c}
\ddot{x}_{1} \\
\ddot{x}_{2} \\
\vdots \\
\ddot{x}_{m}
\end{array}\right)=\left(\begin{array}{c}
\bar{u}_{1} \\
\bar{u}_{2} \\
\vdots \\
\bar{u}_{m}
\end{array}\right) .
$$

Then by combining (9), (10), and (11) it is found that

$$
\left\{\begin{array}{c}
\ddot{z}_{m+1} \\
\vdots \\
\ddot{z}_{n}
\end{array}\right\}=\mathbf{V}^{T}\left\{\begin{array}{c}
f_{1}(\mathbf{z}, \dot{\mathbf{z}}) \\
\vdots \\
f_{n}(\mathbf{z}, \dot{\mathbf{z}})
\end{array}\right\}+\mathbf{V}^{T} \mathbf{G}_{(1: n, 1: m)}\left\{\begin{array}{c}
u_{1} \\
\vdots \\
u_{m}
\end{array}\right\},
$$

so that from (11),

$$
\left\{\begin{array}{c}
\ddot{z}_{m+1} \\
\vdots \\
\ddot{z}_{n}
\end{array}\right\}=\mathbf{V}^{T}\left\{\begin{array}{c}
f_{1}(\mathbf{z}, \dot{\mathbf{z}}) \\
\vdots \\
f_{n}(\mathbf{z}, \dot{\mathbf{z}})
\end{array}\right\}
$$

which ensures uncontrollability of the nonlinear internal dynamics (15).

The stability of the complete system is then determined by the zero dynamics, which are generally nonlinear, obtained by setting to zero in (15) the external coordinates $\left(z_{1}, \ldots, z_{m}\right)$ of the partially linearised system in (13). The equations of the zero dynamics and their stability will be addressed for a specific aeroelastic example in the sequel.

As before, the artificial inputs in (13) may be chosen as a linear combination of the instantaneous displacement and velocity to fulfil a control objective. When the zero dynamics are found to be globally stable, then the desired control behaviour is unaffected by the nonlinearity confined to the internal dynamics.

\section{Aeroservoelastic Model}

The governing equation of aeroservoelastic systems takes the usual form [12] given by

$$
\mathbf{A} \ddot{\mathbf{q}}+(\rho V \mathbf{B}+\mathbf{D}) \dot{\mathbf{q}}+\left(\rho V^{2} \mathbf{C}+\mathbf{E}\right) \mathbf{q}+\mathbf{f}_{\mathrm{nl}}(\mathbf{q})=\mathbf{f}_{\mathrm{ext}},
$$

where A, D, E are the inertia, structural damping, and structural stiffness matrices, respectively, $\mathbf{B}, \mathbf{C}$ are the aerodynamic damping and aerodynamic stiffness matrices, respectively, and $\rho, V$ are air density and velocity, respectively, (the present $\mathbf{B}$ is different from the input distribution matrix, also denoted by $\mathbf{B}$, in Section 1 ). The vector $\mathbf{q}$ contains generalised coordinates describing the motion of the system, whereas the vector $\mathbf{f}_{\text {ext }}$ contains externally applied generalised forcing terms, including control forces and gusts. The nonlinearity is confined to $\mathbf{f}_{\mathrm{nl}}(\mathbf{q})$. Modified aerodynamic strip theory is used to compute the lift and pitch moment and an additional unsteady aerodynamic derivative term is included to account for significant unsteady effects [12], which appears in the matrix $\mathbf{B}$. In practice, the aerodynamic matrices are frequency dependent [12] and the time domain model would need to include aerodynamic states to account for this. The approach used is perfectly adequate for low speed, high aspect ratio wings. The system consists of a wing with an underslung engine attached by a pylon. Aerodynamic forces/moments acting on the pylon-engine arrangement are assumed to be negligible compared to those acting on the wing.

In the analysis presented here the wing deflection is described in terms of two assumed modes and a further generalised coordinate representing the angular displacement of the engine about the axis of the wing. The assumed modes are depicted in Figure 1, which also shows the coordinate system with its origin at the root, leading edge of the wing.

The vertical deflection of the wing $\zeta(x, y)$ is then given by

$$
\zeta=y^{2} q_{1}+y\left(x-x_{f}\right) q_{2}
$$




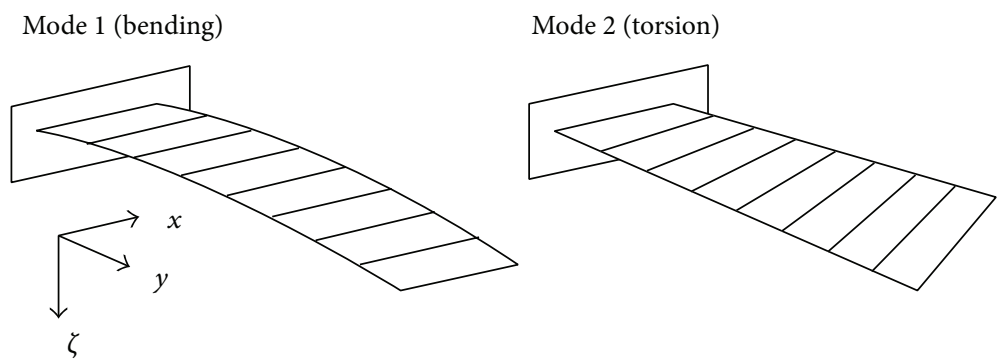

Figure 1: The two deflection patterns assumed for the flexible wing.

where $q_{1}, q_{2}$ are generalised coordinates that quantify the amount of bending and torsion modes present in the overall deflection, and $x_{f}$ is the $x$-coordinate of the wing flexural axis. The pylon-engine is modelled as a rigid body, connected to the wing by a nonlinear stiffness. A sketch of the pylonengine and also the complete wing-pylon-engine system may be found in [13].

The pylon-engine rotational degree of freedom $\vartheta_{\mathrm{pe}}$ is the deflection of the pylon-engine relative to the wing. The absolute rotation $\theta_{\text {pe }}$ may be obtained by adding the wing twist angle at the engine attachment location, $\chi_{2}$, to $\vartheta_{\mathrm{pe}}$. The absolute pylon-engine rotation becomes a coordinate in the assumed-modes domain, $q_{\mathrm{pe}}=\theta_{\mathrm{pe}}$. The coordinate transformation matrix $\mathbf{T}$ is defined by

$$
\left\{\begin{array}{l}
\zeta_{1} \\
\chi_{2} \\
\vartheta_{\mathrm{pe}}
\end{array}\right\}=\left[\begin{array}{ccc}
y_{1}^{2} & 0 & 0 \\
0 & y_{2} & 0 \\
0 & -y_{2} & 1
\end{array}\right]\left\{\begin{array}{l}
q_{1} \\
q_{2} \\
q_{\mathrm{pe}}
\end{array}\right\}
$$

where $\zeta_{1}$ and $\chi_{2}$ denote the vertical displacement at point " 1 " (the intersection of the wing flexural axis with the local $x$-axis at the point of attachment of the pylon to the wing) and the angular wing twist at point " 2 " (the intersection of the quarter-semi-span with the quarter-chord). The $y$ coordinates of points 1 and 2 are identical $\left(y_{1}=y_{2}\right)$. The system matrices, $\mathbf{A}, \mathbf{B}, \mathbf{C}$, and $\mathbf{E}$ are given in terms of the parameters of the wing and pylon-engine system in [13], whereas vectors $\mathbf{f}_{c}$ and $\mathbf{f}_{\mathrm{nl}}$ may be found in Appendix A in this paper. Note that in the present text, $k_{T}, K_{T}$ are used to describe the coefficients of the linear and cubic components of coupling stiffness, respectively, which is different to the notation used in [13].

\section{Numerical Simulation}

The dimensions and parameters chosen for the model are given in [13], with $K_{T}=300 \times k_{T}$. For the flexible wing, the values chosen are based on those used in a numerical example given in [12]. The dimensions and mass of the pylon-engine arrangement are chosen to be representative of a real aircraft (e.g. [14]).

The three degree of freedom aeroservoelastic model is used initially to determine the flutter speed of the linear system. Then, the cubic hardening term, $K_{T}$, is included in the torsional spring connecting the wing to the pylon-engine, and the nonlinear time-domain response to initial conditions is produced at an air speed just above the flutter speed.

The aeroservoelastic matrices are given in general terms in [13] and expressed here in terms of specific parameter values in consistent units (to three significant figures) as

$$
\begin{gathered}
\mathbf{A}_{w}=10^{5}\left[\begin{array}{ccc}
9.49 & 0.0633 & 0 \\
0.0633 & 0.0942 & 0 \\
0 & 0 & 0
\end{array}\right], \\
\mathbf{E}_{w}=10^{8}\left[\begin{array}{ccc}
1.10 & 0 & 0 \\
0 & 0.142 & 0 \\
0 & 0 & 0
\end{array}\right], \\
\mathbf{A}_{\mathrm{pe}}=10^{3}\left[\begin{array}{ccc}
4.76 & 0 & 1.51 \\
0 & 0 & 0 \\
1.51 & 0 & 0.647
\end{array}\right], \\
\mathbf{E}_{\mathrm{pe}}=10^{6}\left[\begin{array}{ccc}
0 & 0 & 0 \\
0 & 1.90 & -0.958 \\
0 & -0.958 & 0.511
\end{array}\right], \\
\mathbf{B}=10^{4}\left[\begin{array}{ccc}
2.98 & 0 & 0 \\
-0.229 & 0.0169 & 0 \\
0 & 0 & 0
\end{array}\right], \\
\mathbf{C}=10^{3}\left[\begin{array}{ccc}
0 & 4.97 & 0 \\
0 & -0.406 & 0 \\
0 & 0 & 0
\end{array}\right] .
\end{gathered}
$$

A simplification in the matrix subscripts previously defined in [13] has been made here. The control force distribution matrix is given (as a function of air speed) by

$$
\begin{aligned}
\mathbf{f}_{c}= & 10^{2}\left[\begin{array}{ccc}
-3.19 V^{2} & -2.89 V^{2} & 0 \\
-0.302 V^{2} & -0.120 V^{2} & -0.0188 \\
0 & 0 & 0.01
\end{array}\right] \\
& \times\left\{\begin{array}{c}
\beta_{1} \\
\beta_{2} \\
T_{\text {pe }}
\end{array}\right\},
\end{aligned}
$$

where $\beta_{1}$ and $\beta_{2}$ are control surface (flap) angles and $T_{\mathrm{pe}}$ is a control torque applied directly to the pylon-engine assembly. The nonlinear internal force is given by

$$
\mathbf{f}_{\mathrm{nl}}=10^{8}\left\{\begin{array}{c}
0 \\
-2.87 \\
1.53
\end{array}\right\} \vartheta^{3}, \quad y_{2}=y_{1}=1.875 \text {. }
$$




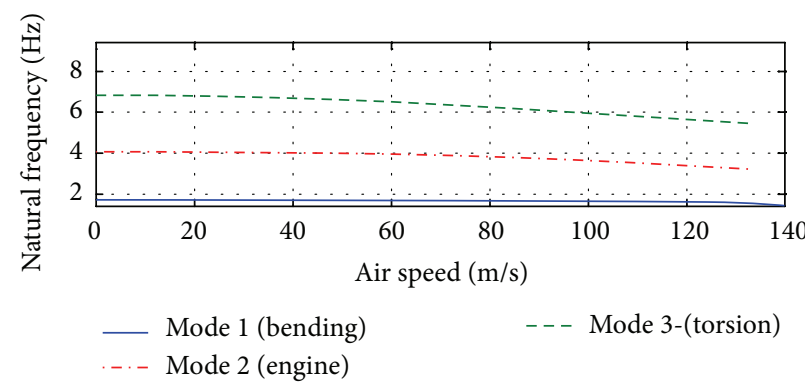

(a)

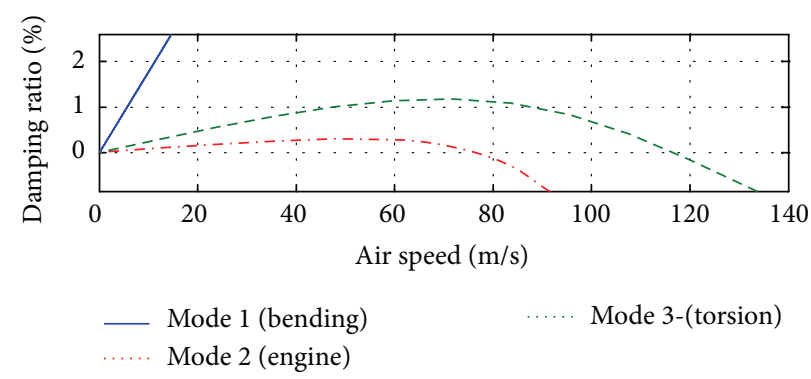

(b)

Figure 2: $V$ - $\omega$ and $V-\zeta$ plots for the wing-pylon-engine model.

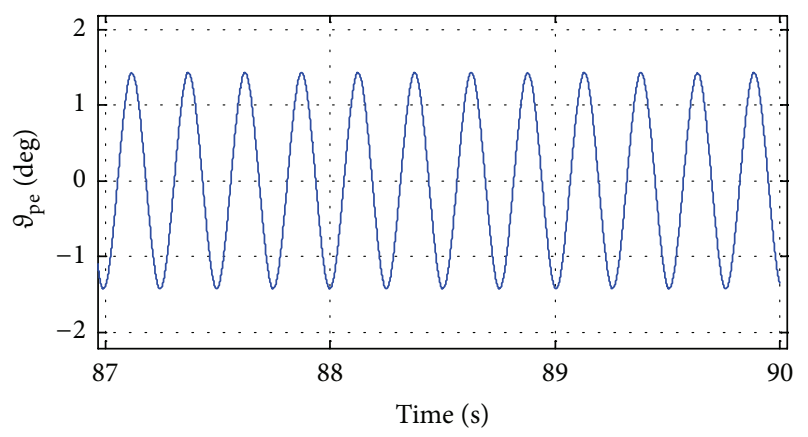

FIGURE 3: Steady-state LCO in $\vartheta_{\text {pe }}$ response.

Further information on the general form of the control and nonlinear spring forces can be found in Appendix A.

\subsection{Uncontrolled Linear System: Natural Frequencies, Damp-} ing and Flutter Speed. The structural modes in the linear model (nonlinearity neglected) occur at $1.71 \mathrm{~Hz}$ (bending), $4.06 \mathrm{~Hz}$ (pylon-engine mode), and $6.83 \mathrm{~Hz}$ (torsional). It is evident from Figure 2 that flutter occurs at an airspeed of $77.6 \mathrm{~ms}^{-1}$, involving coupling of the pylon-engine mode and wing bending modes.

4.2. Nonlinear Time-Domain Response. The nonlinear system is simulated at an airspeed of $80 \mathrm{~ms}^{-1}$, just above the flutter point, under the application of the initial conditions $\zeta_{1}=$ $0.333 \mathrm{~mm}, \chi_{2}=0.00333 \mathrm{rad}$, and $\vartheta_{\mathrm{pe}}=0.05 \mathrm{rad}$. These values have been chosen as they are representative of typical physical displacements one might expect in practice, for the wingpylon-engine system specified in [13]. The resulting response of the system clearly exhibits limit cycle oscillation (LCO). A sample of the response for the $\vartheta_{\text {pe }}$ coordinate is shown in Figure 3.

For comparison, the simulated response for the $\vartheta_{\text {pe }}$ coordinate just below the linear flutter speed, at an airspeed of $75 \mathrm{~ms}^{-1}$, is shown in Figure 4. As expected the response continues to decay and converges to the origin.

In the following section, linearising feedback control is applied to the nonlinear model in the assumed-modes domain. In the examples considered, the control objective is the assignment of the poles of the system. Judicious

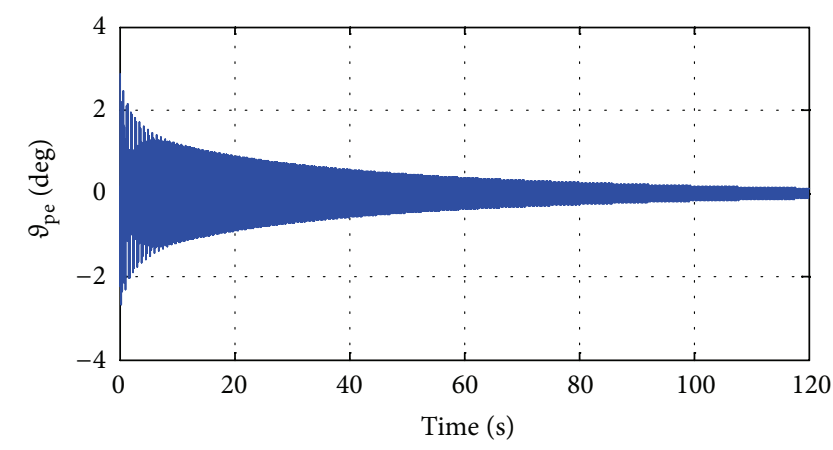

FIgURE 4: Decaying response, just below linear flutter speed.

placement of the poles can be used to increase the flutter speed of the system, thereby increasing the flight envelope. Of course, other control objectives could be used instead. The resulting linearised system consists of the uncoupled single degree of freedom subsystems referred to previously in (5). Two cases are considered: those of 3 inputs and 3 outputs (denoted 3I3O) and of 2 inputs and 2 outputs (denoted 2I2O) which are representative of the analysis in Section 2.

4.3. Feedback Linearisation: $3 I 3 O$ Case. The poles of the system are assigned for the uncoupled linearised system in (5) (by cancellation of the entire open-loop dynamics) and with feedback law (6), so that the natural frequencies and damping values are given by

$$
\begin{array}{rlrl}
\omega_{1} & =0.93 \mathrm{~Hz}, & \xi_{1}=0.01, \\
\omega_{2}=2.9 \mathrm{~Hz}, & \xi_{2}=0.01, \\
\omega_{3}=4.95 \mathrm{~Hz}, & \xi_{3}=0.01,
\end{array}
$$

at an air speed of $80 \mathrm{~ms}^{-1}$. The first mode is predominantly wing bending, the second is pylon-engine deformation and the third is mainly the twisting motion of the wing. With initial conditions the same as the uncontrolled case (Section 4.2), namely $\zeta_{1}=0.333 \mathrm{~mm}, \chi_{2}=$ $0.00333 \mathrm{rad}$, and $\vartheta_{\mathrm{pe}}=0.05 \mathrm{rad}$ and in the absence of any nonlinear parameter error the poles of the linearised system are assigned exactly. The system response now decays to zero as shown in Figure 5, whereas the 


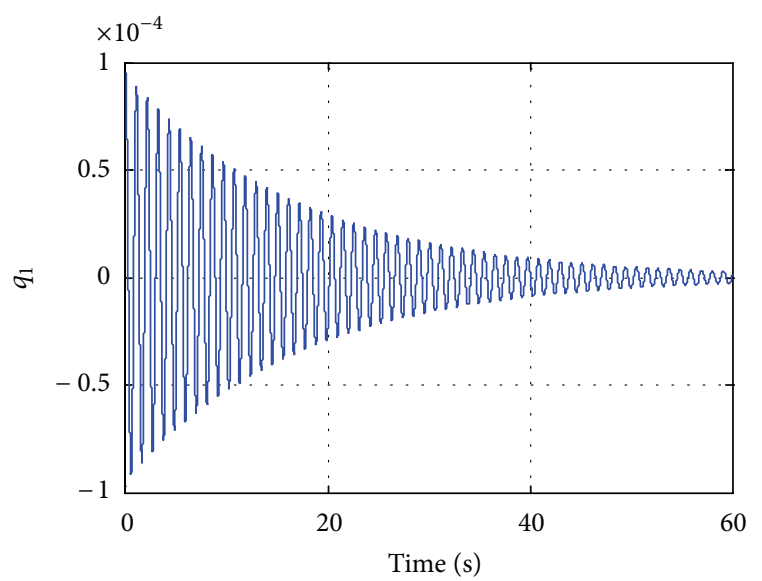

(a)

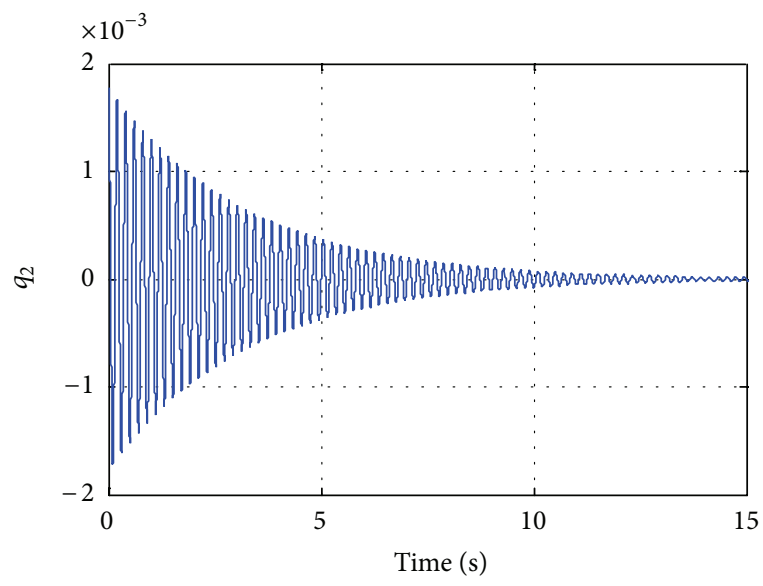

(b)

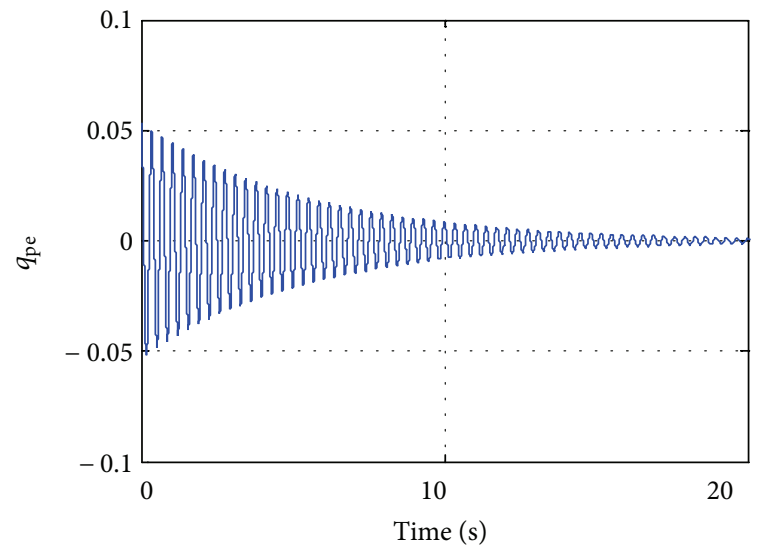

(c)

Figure 5: Feedback linearisation: response at $80 \mathrm{~ms}^{-1}$ (assumedmodes coordinates).

uncontrolled nonlinear system exhibited a limit cycle. The required control surface and actuator inputs are shown in Figure 6, where it can be seen that the input magnitudes are feasible in practice.

Now, suppose the linearising control is chosen to cancel the nonlinearity alone, in which case the linearised equations

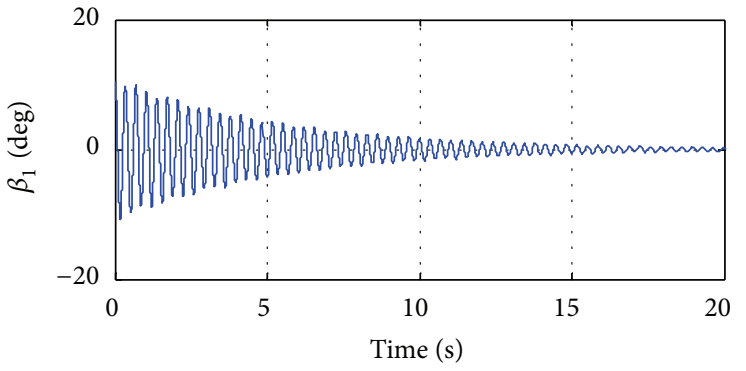

(a)

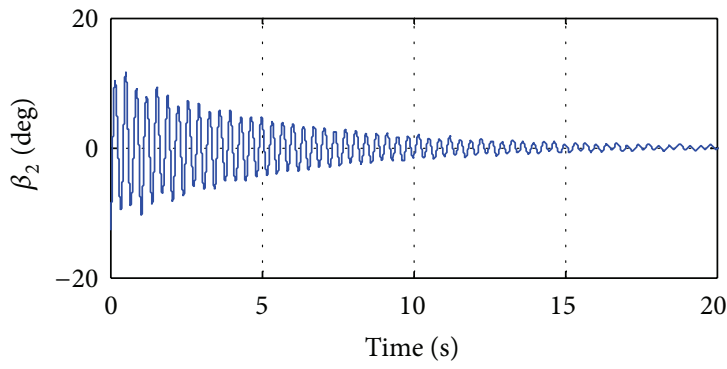

(b)

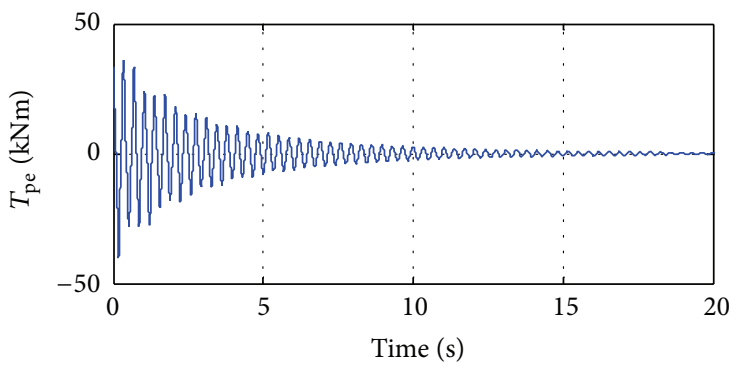

(c)

Figure 6: Control surface deflection angles and actuator torques for exact feedback linearization.

generally remain coupled, resulting in a greater number of control gains in (8) and greater control flexibility as explained in Section 2.1. One is then able to assign eigenvectors corresponding to the assigned poles. The same pole-placement above is now implemented, with the following respective eigenvectors assigned:

$$
\mathbf{v}_{1}=\left\{\begin{array}{lll}
1 & 1 & 0
\end{array}\right\}^{T}, \quad \mathbf{v}_{2}=\left\{\begin{array}{lll}
1 & -1 & 0
\end{array}\right\}^{T}, \quad \mathbf{v}_{3}=\left\{\begin{array}{lll}
0 & 0 & 1
\end{array}\right\}^{T} .
$$

Thus, it is desired to deliberately couple the bending and torsion in the first two modes, whilst leaving the pylon-engine mode decoupled. The resulting closed-loop responses and control inputs are shown in Figures 7 and 8, respectively.

It is evident from the first two plots in Figure 7 that a mixing of modes has occurred, as these two responses now contain multiple harmonics. The third coordinate, however, remains unchanged. This is expected, as the eigenvector assignment commanded that the pylon-engine mode should remain uncoupled. It can also be seen from the first two plots in Figure 8 that the required control surface deflections have increased; it is easier to control the uncoupled modes than the deliberately coupled ones. Thus, in the case of 


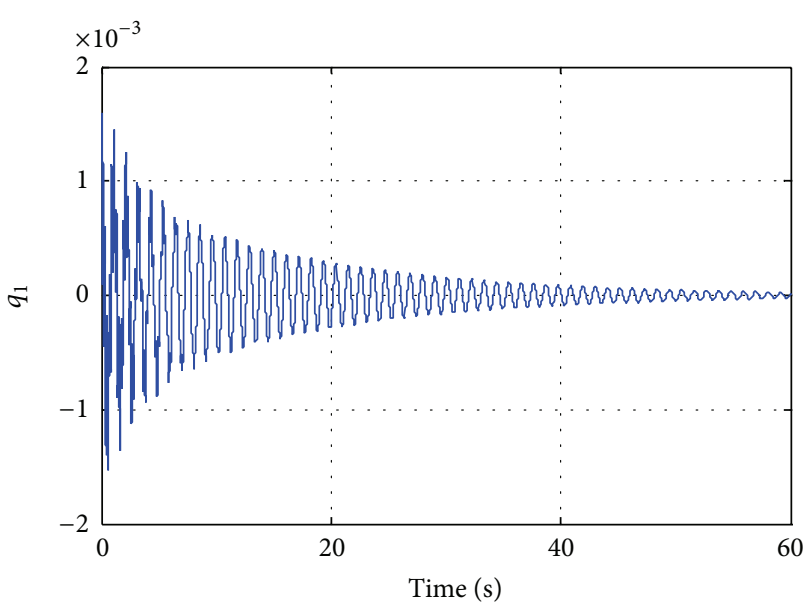

(a)

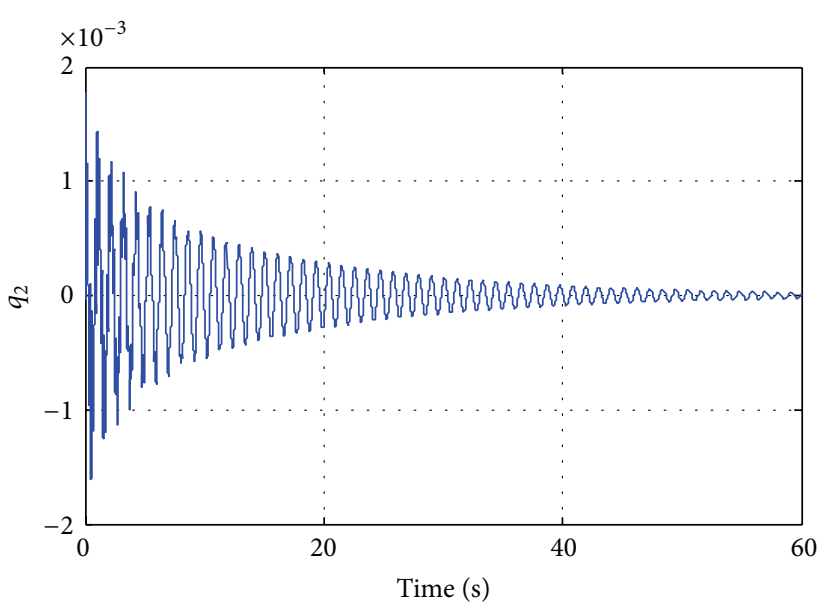

(b)

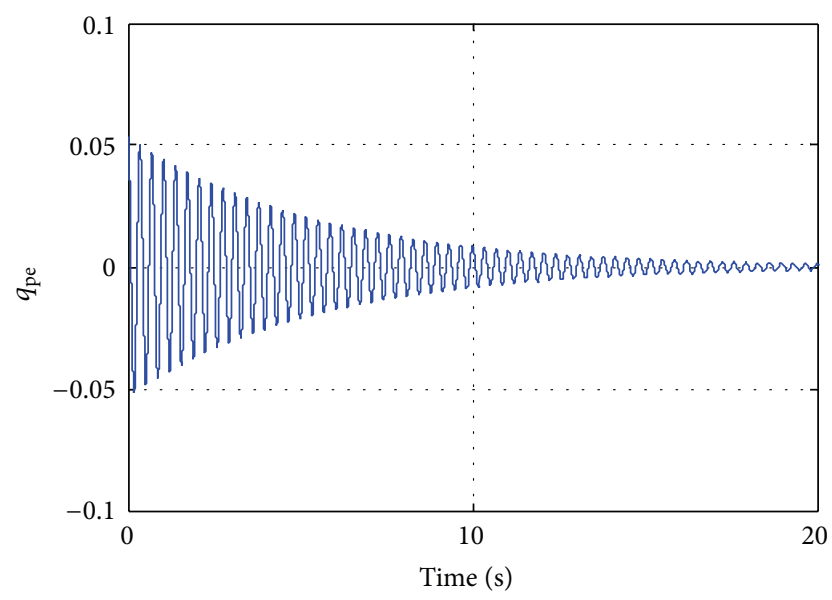

(c)

FIGURE 7: Feedback linearisation with cancellation of nonlinearity only and with eigenvector assignment: response at $80 \mathrm{~ms}^{-1}$ (assumedmodes coordinates).

the three degree of freedom wing-pylon-engine system there is some merit in using feedback linearisation to cancel the entire open loop dynamics despite the loss of control flexibility. For the remainder of this simulation and those appearing later on, only the case where the entire open loop dynamics are cancelled will be considered.

When a $+40 \%$ error in $K_{\mathrm{nl}}$ is incorporated, and the closed-loop response is simulated based on the above feedback parameters and with the same initial conditions, an unstable response sets in from the very beginning, as seen in Figure 9. The nonlinear spring, connected between wing twist and rotation of the pylon-engine assembly, is not capable of suppressing the response (in the assumed-modes coordinates) into a limit cycle. The problem of error in the nonlinear parameter is of practical importance, since it is seldom possible to be precise in estimating the magnitude of nonlinear terms. This problem is addressed in the sequel.

4.4. Feedback Linearisation: $2 I 2 O$ Case. The three degree of freedom system is now considered to be instrumented with only two inputs, trailing edge control-surface (flap) angles $\beta_{1}$ and $\beta_{2}$, and two outputs, discrete wing displacement $\zeta_{1}$ and twist $\chi_{2}$. In this case there is no control torque $T_{\mathrm{pe}}$. The measurement of $\vartheta_{\text {pe }}$ is necessary, but only for determination of $\mathbf{f}(\mathbf{z}, \dot{\mathbf{z}})$. The zero dynamics of the $2 \mathrm{I} 2 \mathrm{O}$ aeroservoelastic problem are given in Appendix B and stabilised by the introduction of structural damping

$$
\mathbf{D}_{\text {mod }}=10^{5}\left[\begin{array}{ccc}
2.05 & 0.0103 & 0.00220 \\
0.0103 & 0.0767 & -0.00282 \\
0.00220 & -0.00282 & 0.00356
\end{array}\right] \text {, }
$$

which corresponds to $1 \%$ of modal damping. The introduction of structural damping increases the flutter speed of the system from $77.6 \mathrm{~ms}^{-1}$ to $95.05 \mathrm{~ms}^{-1}$, and thus the simulation is carried out above the new speed, at $97.5 \mathrm{~ms}^{-1}$. Pole-placement is specified such that

$$
\begin{array}{ll}
\omega_{1}=0.93 \mathrm{~Hz}, & \xi_{1}=0.01, \\
\omega_{2}=4.95 \mathrm{~Hz}, & \xi_{2}=0.01 .
\end{array}
$$




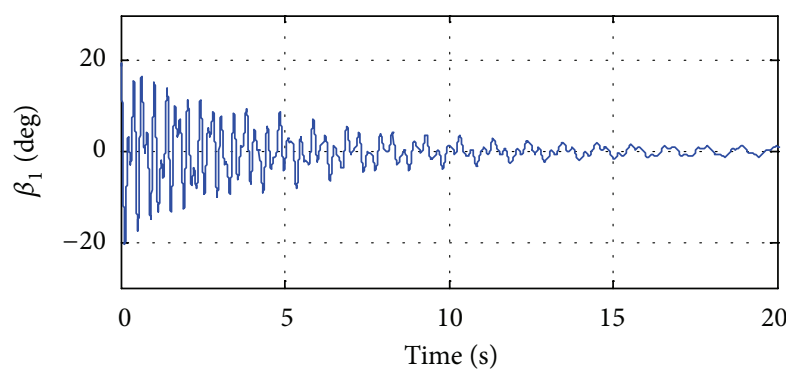

(a)

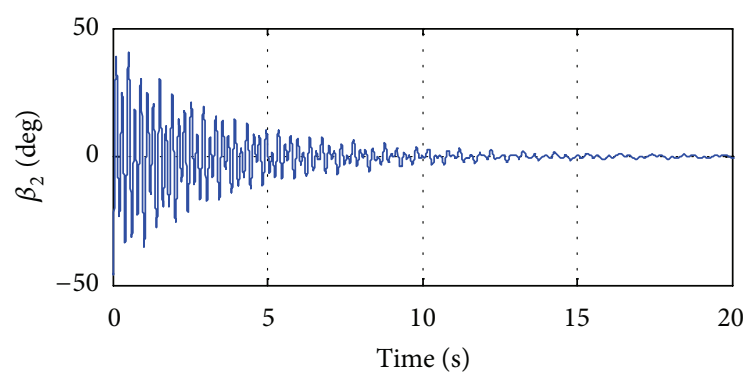

(b)

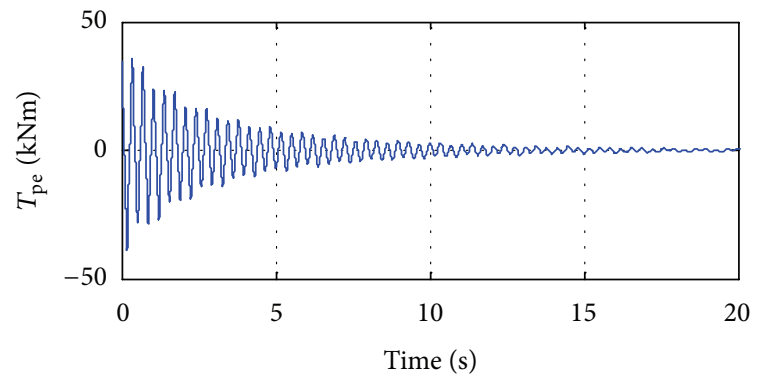

(c)

FIGURE 8: Control surface deflection angles and actuator torques for exact feedback linearisation: cancellation of nonlinearity only, with assignment of eigenvectors.

As before, feedback linearisation successfully places the poles to the desired values and the transient response shown in Figure 10 is obtained.

It is evident from the third subplot in Figure 10 that the coordinate $q_{\mathrm{pe}}$ eventually stabilises because the zero dynamics of the system are stable. When a $+40 \%$ error in $K_{\mathrm{nl}}$ is incorporated and the closed-loop response is simulated based on the same feedback parameters instability does not occur (as in the $3 \mathrm{I} 3 \mathrm{O}$ case) but a degradation in performance is observed in Figure 11 where it is seen that the control fails to eliminate an LCO.

\section{Treatment of Nonlinear Parameter Error: Adaptive Feedback Linearisation}

The treatment of error in the numerical value of a nonlinear parameter is carried out using adaptive feedback linearisation, which makes use of Lyapunov stability theory. It results in an updating rule for the erroneous parameter, which evolves with time. This approach ensures that the system remains stable; the original control objectives might be compromised because of evolution of the nonlinear parameter estimate. This might be acceptable in many cases depending upon engineering judgment.

An erroneous estimate, $\mathbf{f}_{\mathrm{n}}^{\prime}$, is now assumed in place of the true nonlinear force vector, $\mathbf{f}_{\mathrm{nl}}$. The corresponding erroneous input is then determined, by comparison with (2) and (4), as

$$
\mathbf{u}^{\prime}=\mathbf{G}^{-1}\left(\overline{\mathbf{u}}-\mathbf{f}^{\prime}(\mathbf{x})\right), \quad \mathbf{f}^{\prime}(\mathbf{x})=\Psi \mathbf{x}+\Phi \dot{\mathbf{x}}+\boldsymbol{\Omega} \mathbf{f}_{\mathrm{nl}}^{\prime},
$$

and substituting (26) into (2) leads to

$$
\ddot{\mathbf{x}}=\overline{\mathbf{u}}+\Omega \boldsymbol{\varepsilon}, \quad \boldsymbol{\varepsilon}=\mathrm{f}_{\mathrm{nl}}-\mathbf{f}_{\mathrm{nl}}^{\prime} .
$$

Recall (6) from Section 2.1 earlier. This may be combined into a single equation

$$
\overline{\mathbf{u}}=\Gamma\left\{\begin{array}{l}
\mathbf{x} \\
\dot{\mathbf{x}}
\end{array}\right\}, \quad \Gamma \in \mathbb{R}^{n \times 2 n} .
$$

The artificial inputs may be expressed in terms of the state variables, $\mathbf{x}$, by using the above equation together with the coordinate transformation

$$
\mathbf{z}=\mathbf{T}_{z x} \mathbf{x}
$$

defined in (10) and (11).

Thus,

$$
\overline{\mathbf{u}}=\boldsymbol{\Gamma}^{\prime}\left\{\begin{array}{l}
\mathbf{x} \\
\dot{\mathbf{x}}
\end{array}\right\}=\left[\begin{array}{ll}
\Gamma_{1}^{\prime} & \Gamma_{2}^{\prime}
\end{array}\right]\left\{\begin{array}{l}
\mathbf{x} \\
\dot{\mathbf{x}}
\end{array}\right\}, \quad \Gamma^{\prime}=\boldsymbol{\Gamma}_{z}\left[\begin{array}{cc}
\mathbf{T}_{z x} & \mathbf{0} \\
\mathbf{0} & \mathbf{T}_{z x}
\end{array}\right],
$$

and the second-order equation of the closed-loop system is then cast as

$$
\ddot{\mathbf{x}}=\Gamma_{1}^{\prime} \mathbf{x}+\Gamma_{2}^{\prime} \dot{\mathbf{x}}+\mathbf{\Omega \varepsilon} .
$$

Equation (31) shows that the nonlinear parameter error results in an input to the closed-loop system. It may be readily shown, for the $3 \mathrm{I} 3 \mathrm{O}$ system, that

$$
\Gamma_{1, z}^{\prime}=-\left[\begin{array}{lll}
\varsigma_{1} & & \\
& \varsigma_{2} & \\
& & \varsigma_{2}
\end{array}\right], \quad \Gamma_{2, z}^{\prime}=-\left[\begin{array}{lll}
\nu_{1} & & \\
& \nu_{2} & \\
& & \nu_{2}
\end{array}\right] .
$$

The system is then represented in state-space as

$$
\begin{gathered}
\dot{\overline{\mathbf{z}}}=\overline{\mathrm{A}} \overline{\mathbf{z}}+\Delta K_{T} \vartheta_{\mathrm{pe}}^{3} \overline{\mathbf{B}} \boldsymbol{\Omega}\left(\mathbf{T}_{(3,:)}\right)^{T}, \\
\overline{\mathbf{A}}=\left[\begin{array}{cc}
\mathbf{0} & \mathbf{I} \\
\boldsymbol{\Gamma}_{1, z}^{\prime} & \boldsymbol{\Gamma}_{2, z}^{\prime}
\end{array}\right], \\
\overline{\mathbf{B}}=\left[\begin{array}{l}
\mathbf{0} \\
\mathbf{I}
\end{array}\right], \quad \overline{\mathbf{z}}=\left\{\begin{array}{l}
\mathbf{z} \\
\dot{\mathbf{z}}
\end{array}\right\},
\end{gathered}
$$

where $\mathbf{T}$ is defined in (18) and where the erroneous nonlinear parameter is given by

$$
\begin{gathered}
K_{T}^{\prime}=K_{T}+\Delta K_{T}, \\
\boldsymbol{\varepsilon}=\Delta K_{T} \vartheta_{\mathrm{pe}}^{3} \boldsymbol{\Omega}\left(\mathbf{T}_{(3 ;:)}\right)^{T} .
\end{gathered}
$$




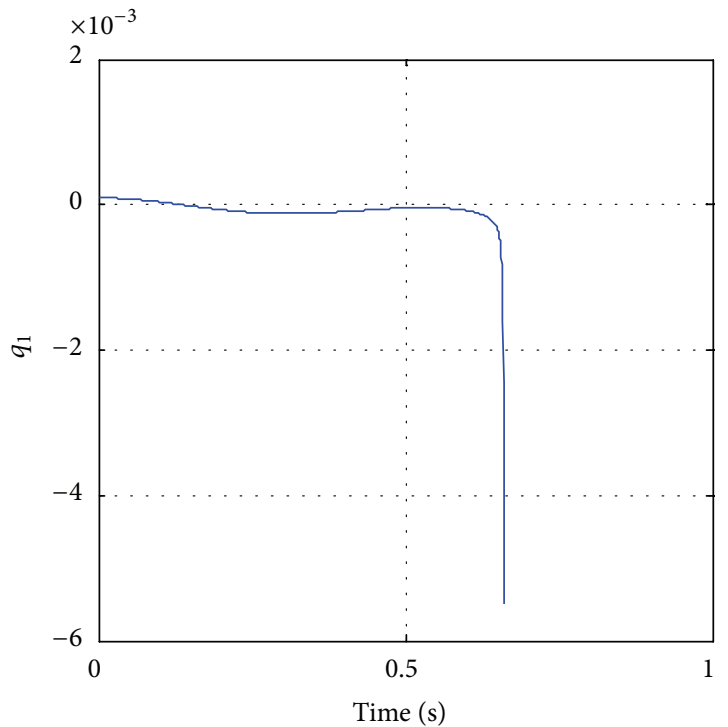

(a)

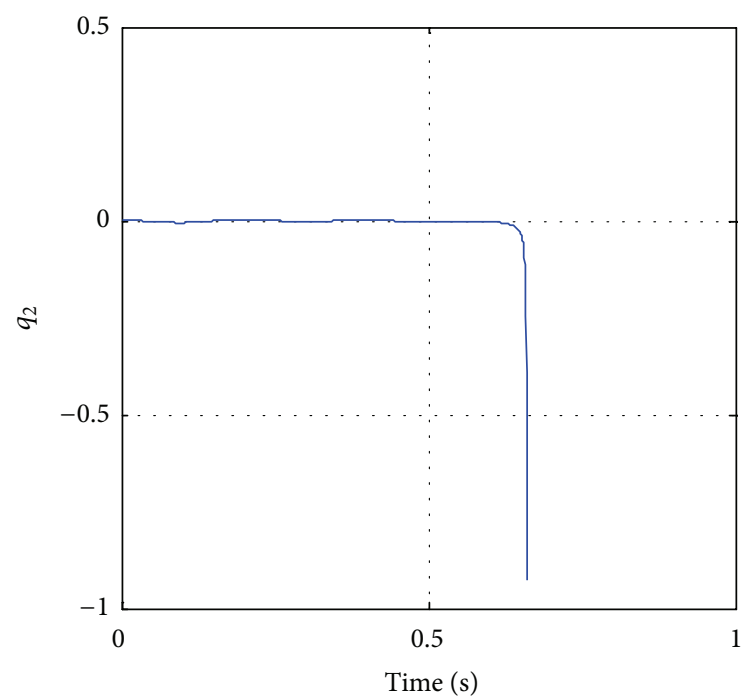

(b)

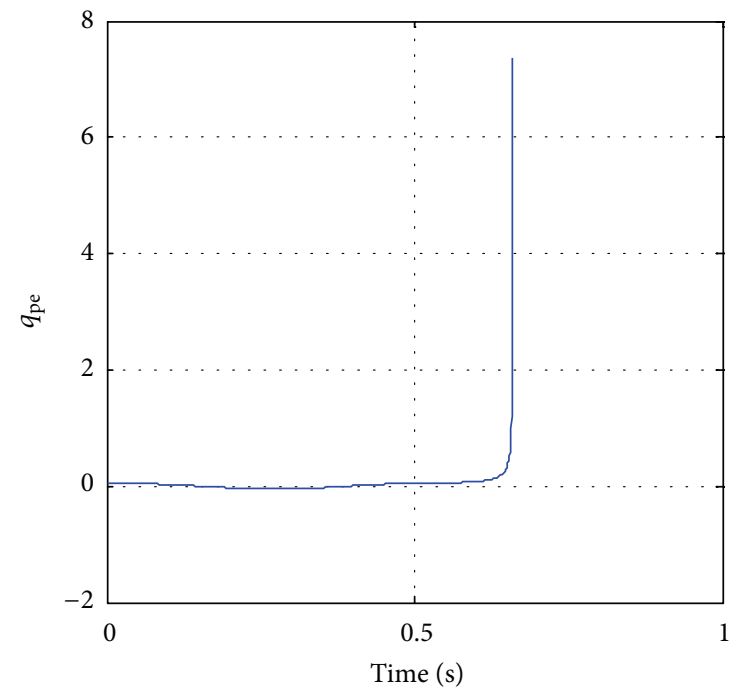

(c)

FIGURE 9: Feedback-linearisation with nonlinear parameter error: response at $80 \mathrm{~ms}^{-1}$.

It is conceivable that this additional (unknown) input may potentially destabilise the system, or at least degrade the control performance. This possibility may be eliminated by accounting for the nonlinearity errors using an adaptive algorithm. Such an algorithm seeks to guarantee asymptotic stability of the closed-loop response.

A scalar Lyapunov function $V$ in $\overline{\mathbf{z}}, \Delta K_{T}$ may be defined, such that asymptotic stability of the closed-loop system is guaranteed by ensuring that $V>0$ and its time-derivative $\dot{V}<0$ [15]. Such a function may be used as a basis for computing a parameter update law. The Lyapunov function,

$$
V\left(\overline{\mathbf{z}}, \Delta K_{T}\right)=\overline{\mathbf{z}}^{T} \mathbf{P} \overline{\mathbf{z}}+\Delta K_{T}^{2}>0
$$

is considered, where $\mathbf{P}=\mathbf{P}^{T}>0$. Differentiating the above equation with respect to time, one obtains

$$
\dot{V}\left(\overline{\mathbf{z}}, \Delta K_{T}\right)=\dot{\overline{\mathbf{z}}}^{T}\left(\mathbf{P}+\mathbf{P}^{T}\right) \overline{\mathbf{z}}+2 \Delta K_{T} \frac{\mathrm{d}\left(\Delta K_{T}\right)}{\mathrm{d} t} .
$$

Now, by combining (33) and (37) and expanding and rearranging

$$
\begin{aligned}
\dot{V}\left(\overline{\mathbf{z}}, \Delta K_{T}\right)= & \overline{\mathbf{z}}^{T}\left(\overline{\mathbf{A}}^{T} \mathbf{P}+\mathbf{P} \overline{\mathbf{A}}\right) \overline{\mathbf{z}} \\
& +2 \Delta K_{T}\left(\frac{\mathrm{d}\left(\Delta K_{T}\right)}{\mathrm{d} t}+\vartheta_{\mathrm{pe}}^{3} \mathbf{T}_{(3,:)} \boldsymbol{\Omega}^{T} \overline{\mathbf{B}}^{T} \mathbf{P} \overline{\mathbf{z}}\right),
\end{aligned}
$$




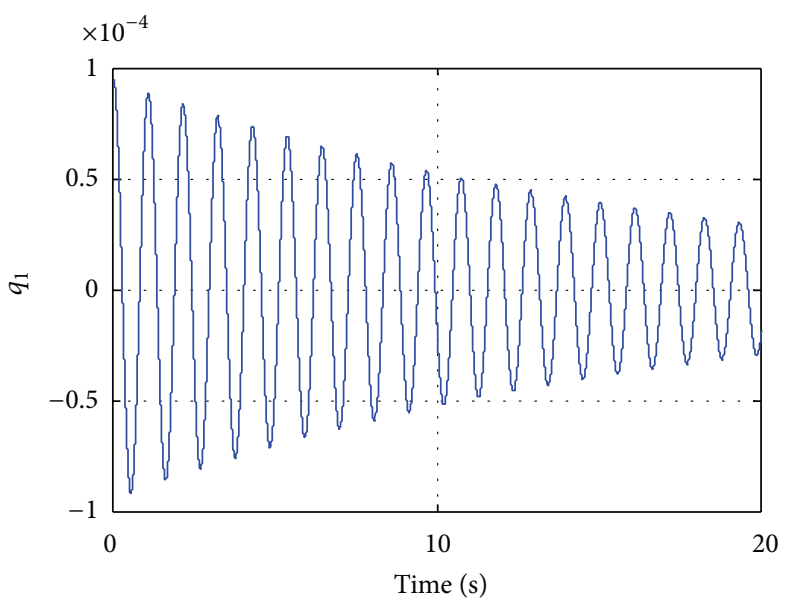

(a)

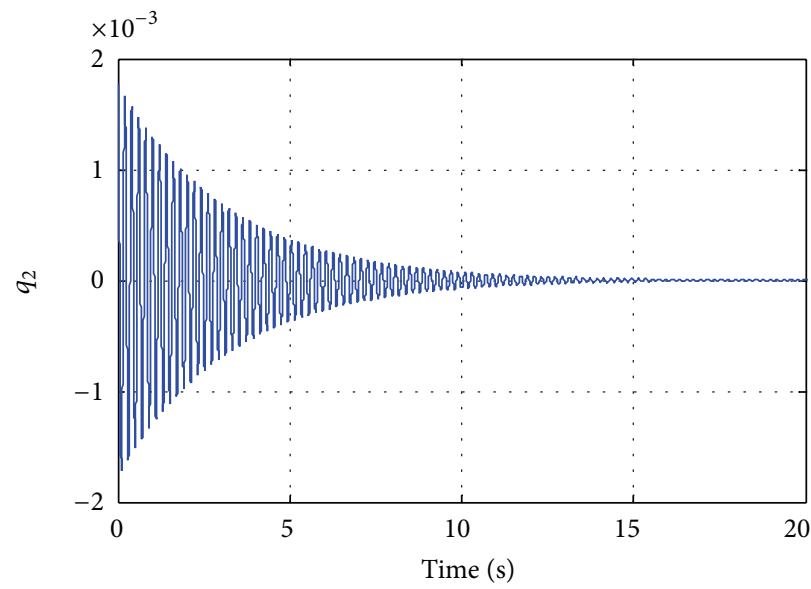

(b)

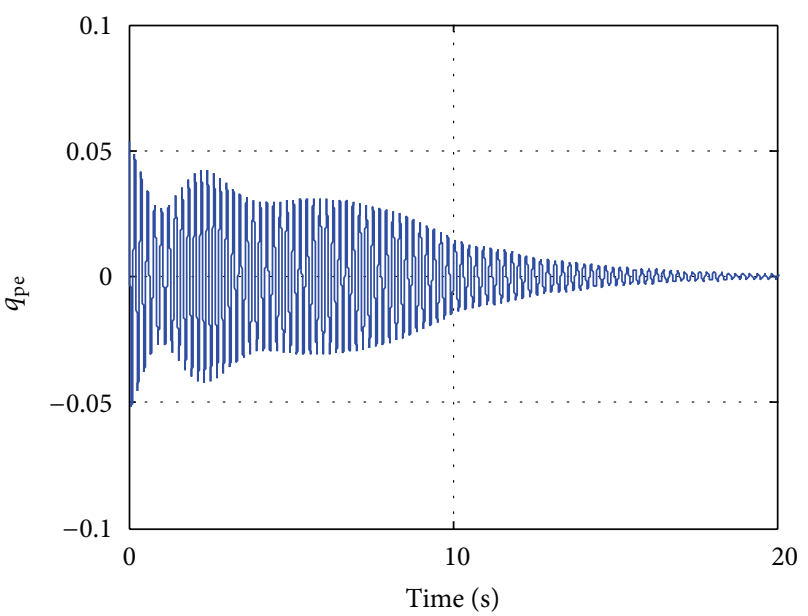

(c)

FiguRE 10: Feedback-linearisation: response at $97.5 \mathrm{~ms}^{-1}$ (assumed-modes coordinates).

it becomes evident that

$$
\frac{\mathrm{d}\left(\Delta K_{T}\right)}{\mathrm{d} t}=-\vartheta_{\mathrm{pe}}^{3} \mathbf{T}_{(3,:)} \boldsymbol{\Omega}^{T} \overline{\mathbf{B}}^{T} \mathbf{P} \overline{\mathbf{z}}
$$

eliminates the second term on the right-hand-side of (38), resulting in

$$
\dot{V}\left(\overline{\mathbf{z}}, \Delta K_{T}\right)=\overline{\mathbf{z}}^{T}\left(\overline{\mathbf{A}}^{T} \mathbf{P}+\mathbf{P} \overline{\mathbf{A}}\right) \overline{\mathbf{z}}
$$

Now, from the definition of $\Delta K_{T}$ in (34) and knowing that the actual nonlinear parameter $K_{T}$ is constant, it is seen that

$$
\frac{\mathrm{d}\left(\Delta K_{T}\right)}{\mathrm{d} t}=\frac{\mathrm{d}\left(K_{T}\right)}{\mathrm{d} t}-\frac{\mathrm{d}\left(K_{T}^{\prime}\right)}{\mathrm{d} t}=-\frac{\mathrm{d}\left(K_{T}^{\prime}\right)}{\mathrm{d} t} .
$$

Combining (41) and (39), an update law for $K_{T}^{\prime}$ corresponding to the latter equation is obtained as

$$
\frac{\mathrm{d}\left(K_{T}^{\prime}\right)}{\mathrm{d} t}=\vartheta_{\mathrm{pe}}^{3} \mathbf{T}_{(3,:)} \boldsymbol{\Omega}^{T} \overline{\mathbf{B}}^{T} \mathbf{P} \overline{\mathbf{z}}
$$

Thus, the initially assumed value $K_{T}^{\prime}$ of the nonlinear parameter is continually updated at each time step. Effectively, this increases the dimension of the state vector by 1 , as $K_{T}^{\prime}$ becomes part of the system state. Now, choosing $\mathbf{P}$ such that for arbitrary $\mathbf{Q}>0$,

$$
\mathbf{Q}=-\left(\overline{\mathbf{A}}^{T} \mathbf{P}+\mathbf{P} \overline{\mathbf{A}}\right),
$$

it becomes clear that by substituting the above equation into (40), $\dot{V}$ is rendered negative-definite, as required.

For the $2 \mathrm{I} 2 \mathrm{O}$ case, an identical approach may be followed to obtain similar expressions. The definition of $\overline{\mathbf{z}}$ changes to $\overline{\mathbf{z}}=\left\{\begin{array}{ll}\mathbf{z}_{(1: 2)} & \dot{\mathbf{z}}_{(1: 2)}\end{array}\right\}^{T}$, and the parameter update rate becomes

$$
\frac{\mathrm{d}\left(K_{T}^{\prime}\right)}{\mathrm{d} t}=\vartheta_{\mathrm{pe}}^{3} \mathbf{T}_{(3,:)} \boldsymbol{\Omega}_{(1: 2,:)}^{T} \overline{\mathbf{B}}^{T} \mathbf{P} \overline{\mathbf{z}} .
$$

The matrices $\overline{\mathbf{A}}$ and $\overline{\mathbf{B}}$ become

$$
\overline{\mathbf{A}}=\left[\begin{array}{cc}
\mathbf{0} & \mathbf{I}_{2 \times 2} \\
\boldsymbol{\Gamma}_{1, z}^{\prime}(1: 2,1: 2) & \boldsymbol{\Gamma}_{2, z_{(1: 2,1: 2)}^{\prime}}^{\prime}
\end{array}\right], \quad \overline{\mathbf{B}}=\left[\begin{array}{c}
\mathbf{0} \\
\mathbf{I}_{2 \times 2}
\end{array}\right] .
$$




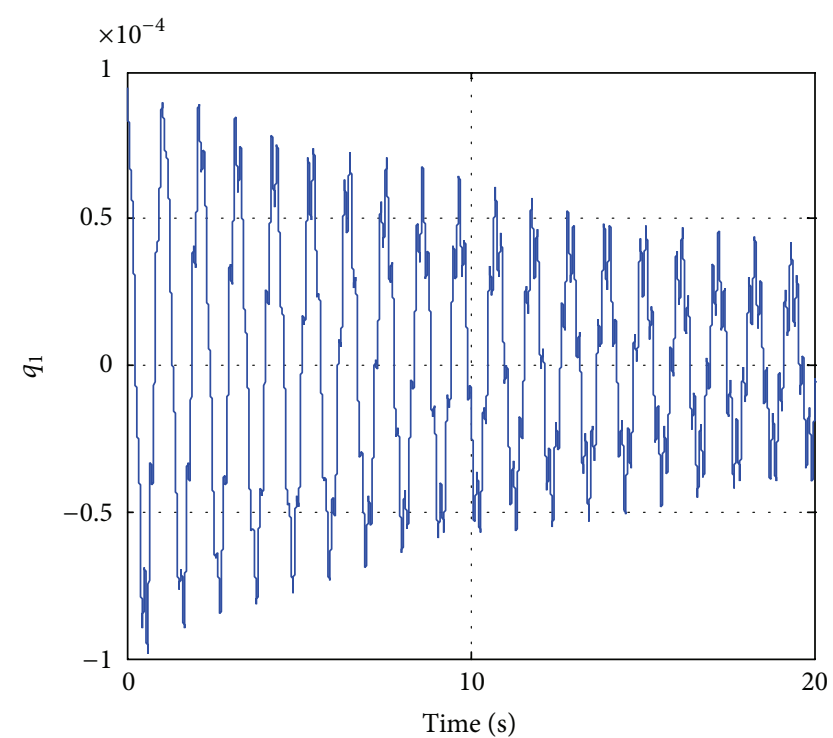

(a)

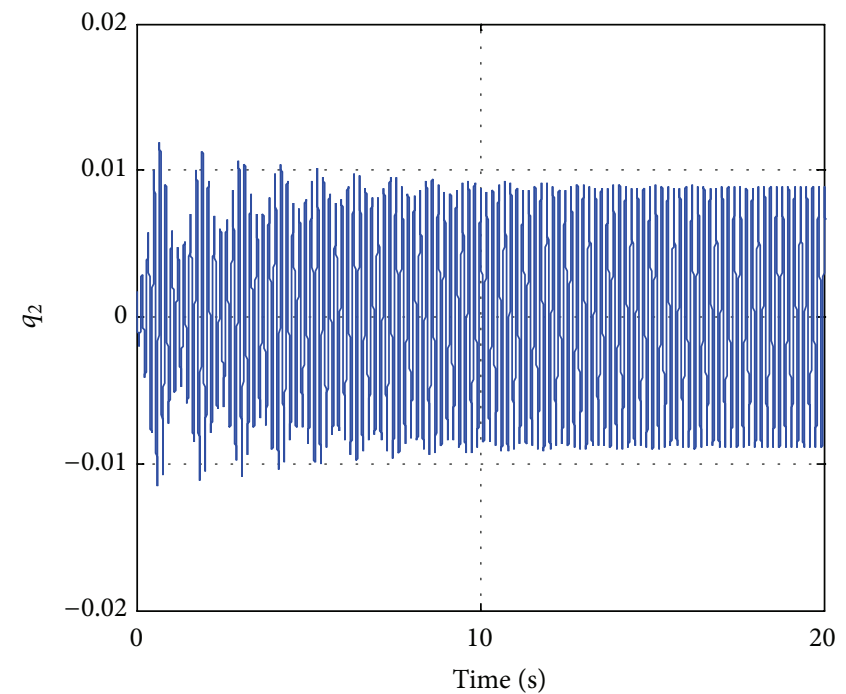

(b)

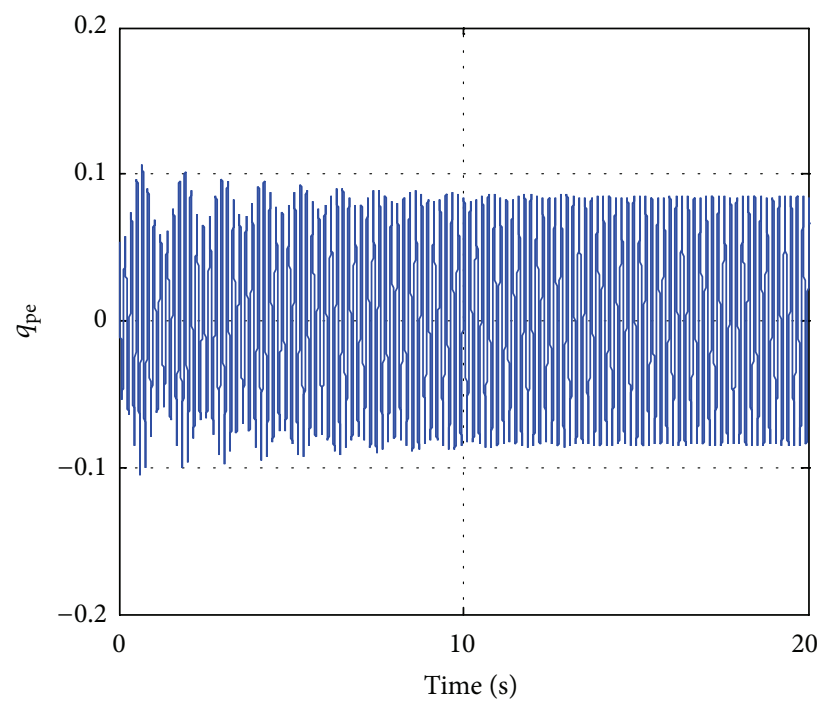

(c)

FIGURE 11: Feedback-linearisation with nonlinear parameter error: response at $97.5 \mathrm{~ms}^{-1}$.

An important consideration in the $2 \mathrm{I} 2 \mathrm{O}$ case is the asymptotic stability of the zero dynamics, considered in Appendix B, which is required for the adaptive scheme to work. For the particular 2I2O configuration considered, the presence of structural damping ensures stability of the zero dynamics, thus enabling application of the adaptive method.

Example: Stabilisation of the Aeroelastic System Using Adaptive Feedback Linearisation. The instability shown in Figure 9 may be avoided altogether by implementing the adaptive controller described in Section 5 above. In the $3 \mathrm{I} 3 \mathrm{O}$ case, the resulting controlled response, for the same initial conditions, is shown in Figure 12.

A comparison of Figure 12 with Figure 5 shows that the controlled responses are similar, but not identical. Although adaptive feedback linearisation destroys the original pole-placement, it can be seen that the controlled response is stable. In fact, the adaptive controller successfully drives the responses to zero for values of $\left|\vartheta_{\text {pe }}\right|_{\max }$ (set as an initial condition) up to around $0.0735 \mathrm{rad}$. The required control in this case is accomplished through achievable control surface deflection angles $\left(\approx 11^{\circ}\right)$ and actuator torque magnitude $(55 \mathrm{kNm})$.

In the case of the $2 \mathrm{I} 2 \mathrm{O}$ system, the system response with adaptation shown in Figure 13 should be compared to that in Figure 11, for feedback linearisation without adaptation and the same error in the cubic stiffness parameter. The degradation of the system to LCO is completely removed in Figure 13.

It can also be seen that the controlled responses, as well as the response of the uncontrolled coordinate converge to zero when the adaptive controller is implemented. In fact, 


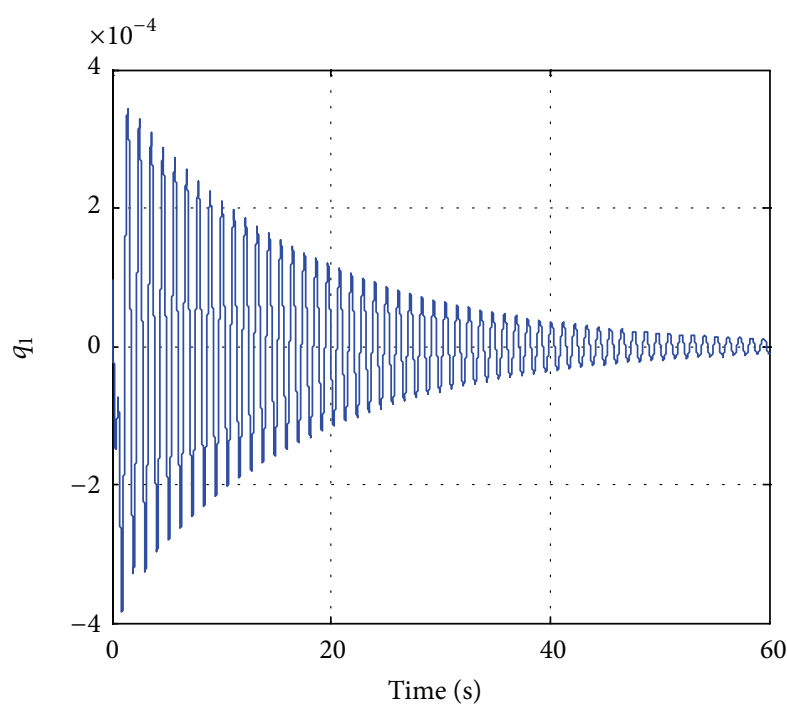

(a)

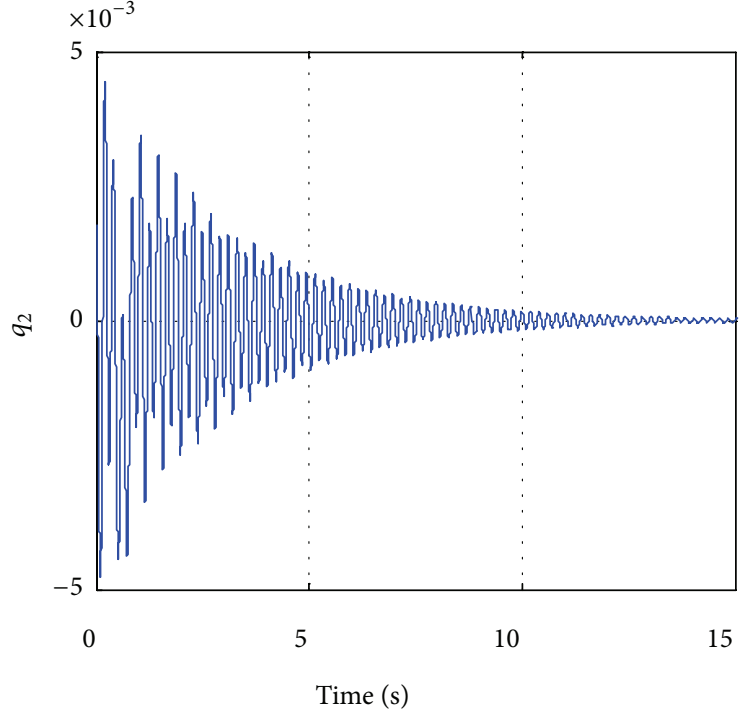

(b)

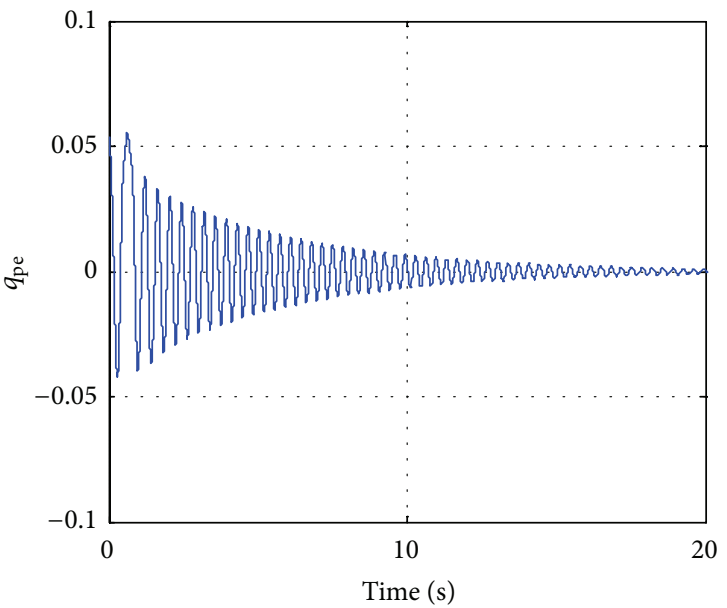

(c)

FIgURE 12: 3I3O system with adaptive feedback linearisation: response at $80 \mathrm{~ms}^{-1}$.

this convergence takes place rapidly. When converted to the physical domain, the resulting magnitudes of the coordinates occur within acceptable limits (maximum values of $0.33 \mathrm{~mm}$, $0.24^{\circ}, 2.92^{\circ}$ for $\zeta_{1}, \chi_{2}, \vartheta_{\text {pe }}$, resp.). As before, it is found that the control surface deflections required to achieve the responses shown in Figure 13 are feasible $\left(\beta_{1}, \beta_{2} \approx 30^{\circ}\right)$.

\section{Conclusions}

Nonlinear systems are ubiquitous in vibrations engineering and aeroelasticity, but the analysis is mathematically intricate and complicated. The paper presents the feedback linearisation methodology, whereby a nonlinear system is rendered linear by means of active control. Having neutralised the nonlinearity, the system may be treated using well-understood linear analysis methods such as modal decomposition, which generally cannot be applied to nonlinear systems directly.
The technique is formulated using the second-order representation of elastomechanical and aeroelastic systems with stiffness nonlinearity, familiar to the vibrations community. This has certain advantages over the conventional state-space formulation in that repeated output differentiation, usually described using the Lie algebra notation, is unnecessary. The purpose of the linearising controller is to cancel the nonlinearity completely and therefore it results in a truly linear system, rather than linearisation about an operating point limited to small perturbations, or quasi-linearisation as with describing functions. The controller may be designed to cancel the nonlinearity only or to cancel the complete openloop dynamics. In the former case there is shown to be greater control flexibility, but the latter case is found to have some merit in the particular example considered of a flexible wingpylon-engine system with decoupled bending, torsional, and pylon-engine modes. 


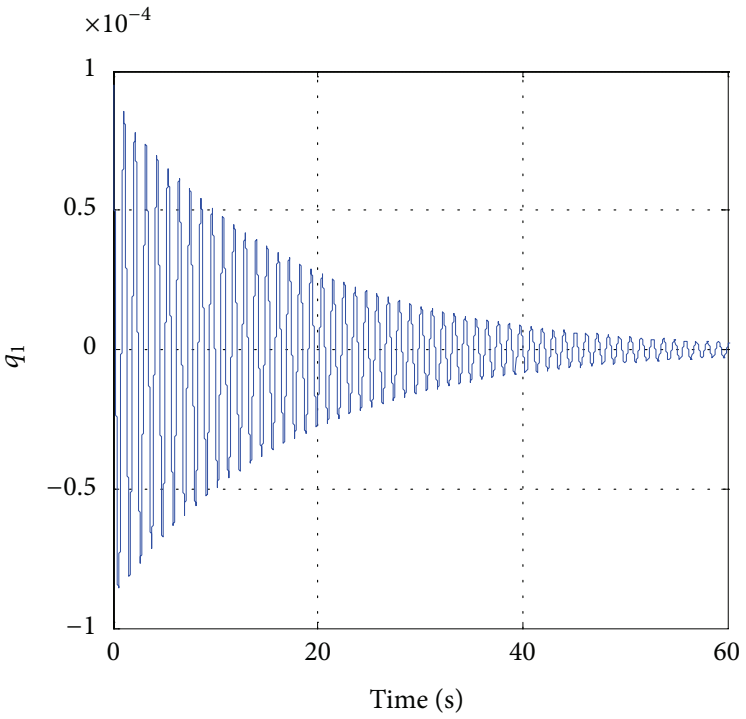

(a)

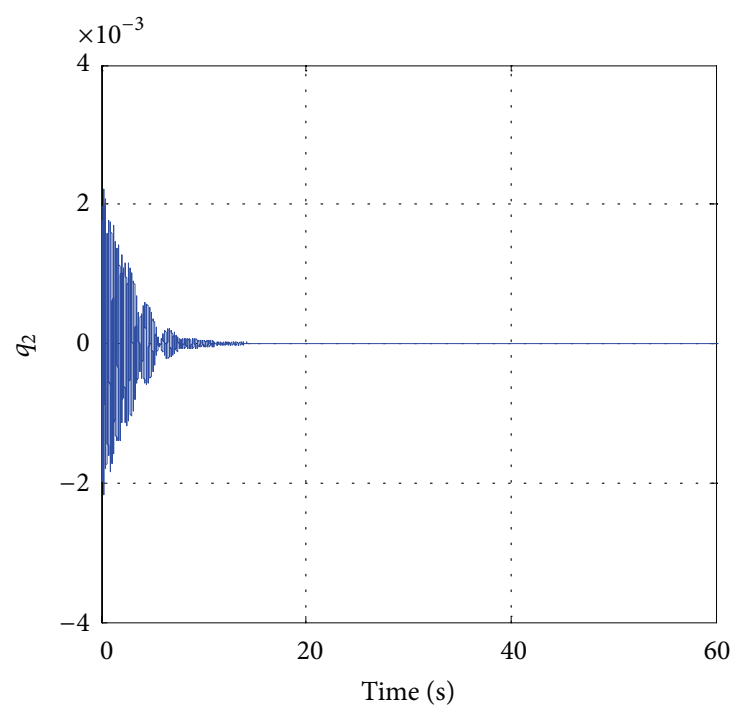

(b)

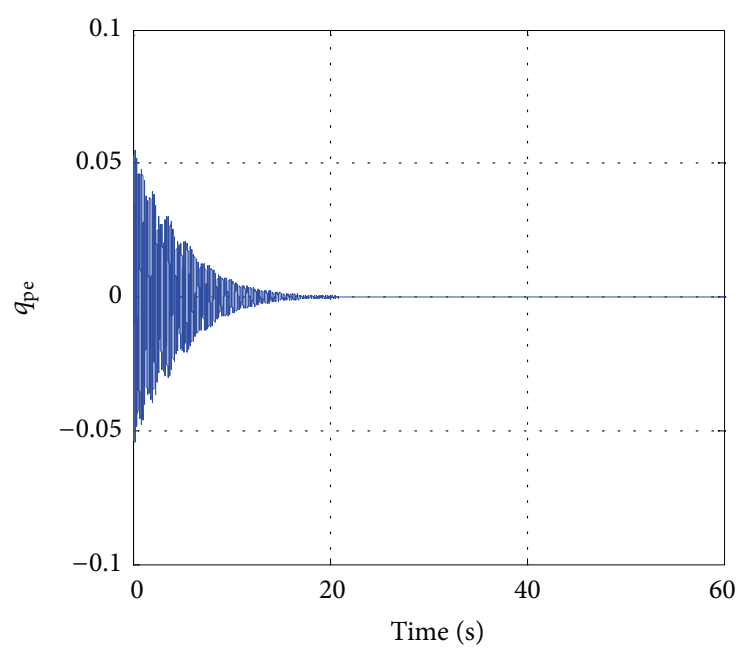

(c)

FIGURE 13: 2I2O system with feedback linearisation: response at $97.5 \mathrm{~ms}^{-1}$.

Exact feedback linearisation requires knowledge of the nonlinearity and when every degree of freedom of the system is available for measurement (and actuation) and then linearisation may be achieved completely. When fewer than the full set of degrees of freedom is available for measurement and actuation, then the system can generally be partitioned into independent linear and nonlinear subsystems, with the dimension of the latter being the difference between the number of degrees of freedom and the number of sensors and actuators. If the nonlinear subsystem is stable, then the dynamics of the linear subsystem may be controlled as required. The problem of an imprecisely known nonlinear term was addressed using adaptive feedback linearisation, resulting in a parameter update rule that evolves in time to ensure stability. It requires an additional state variable to do this and is likely to be more expensive than using feedback linearisation without adaptivity.

Feedback linearisation techniques were illustrated using a three degree of freedom aeroelastic model consisting of a flexible wing and a rigid pylon-engine system attached to the wing via a nonlinear torsional spring with pole placement of the fully linearised and partially linearised system corresponding to the measurement (and actuation) at three degrees of freedom (3I3O) and two degrees of freedom (2I2O) respectively. The parameters of the system were chosen carefully to represent a real aircraft. Adaptive linearisation was applied successfully to compensate for a nonlinear parameter error in the case of $3 \mathrm{I} 3 \mathrm{O}$, and in the $2 \mathrm{I} 2 \mathrm{O}$ case the nonlinear subsystem was found to be stable. The magnitudes of the aileron deflection angles were physically realisable. 


\section{Appendices}

\section{A. Control Forces and Nonlinear Spring Forces}

Control forces are applied to the wing-pylon-engine system by means of control surfaces. It is assumed in this work that two control surfaces (different from [14]) are available, the first (closest to the wing root) spanning $85 \%$ of the length of the wing and the second spanning the remaining length (the contribution of the control surfaces to the dynamics of the overall system is neglected). The widths of the first and second control surfaces are assumed to be $20 \%$ and $33.33 \%$ of the chord length, respectively. These particular dimensions have been chosen so as to optimise the distribution of work performed by each control surface. In addition to the ailerons, it is assumed that a separate actuator is available to apply a torque $T_{\text {pe }}$ directly on the engine rotational degree of freedom. The forcing vector is found to be

$$
\begin{aligned}
\mathbf{f}_{c}= & {\left[\begin{array}{ccc}
-\frac{1}{6} \widetilde{r} \alpha^{3} a_{C, 1} s_{w} & -\frac{1}{6} \widetilde{r}\left(1-\alpha^{3}\right) a_{C, 2} s_{w} & 0 \\
\frac{1}{4} \widetilde{r} \alpha^{2} b_{C, 1} c_{w} & \frac{1}{4} \widetilde{r}\left(1-\alpha^{2}\right) b_{C, 2} c_{w} & -y_{2} \\
0 & 0 & 1
\end{array}\right] } \\
& \times\left\{\begin{array}{c}
\beta_{1} \\
\beta_{2} \\
T_{\mathrm{pe}}
\end{array}\right\}=\mathbf{B u},
\end{aligned}
$$

where $\tilde{r}=\rho V^{2} c_{w} s_{w}^{2}, \alpha=0.85$ and each surface will have its own deflection angle $\beta_{1}, \beta_{2}$ and set of aerodynamic parameters $a_{C}, b_{C}$, which are the rates of change of lift coefficient and moment coefficient, respectively, with respect to control surface deflection angle.

A cubic hardening nonlinearity is assumed in the torsional spring connecting the pylon-engine to the wing. The nonlinear force developed in the spring may be expressed as

$$
f_{\mathrm{nl}}=K_{T} \vartheta_{\mathrm{pe}}^{3}
$$

where $K_{T}$ is the stiffness coefficient of the cubic nonlinearity. The overall nonlinear force vector then takes the form

$$
\mathbf{f}_{\mathrm{nl}}=\mathbf{T}^{T}\left(\begin{array}{c}
0 \\
0 \\
f_{\mathrm{nl}}
\end{array}\right)=\left(\begin{array}{c}
0 \\
-y_{2} f_{\mathrm{nl}} \\
f_{\mathrm{nl}}
\end{array}\right)
$$

with $\mathbf{T}$ defined in (18).

\section{B. Zero Dynamics Expressions (2I20 Case)}

In the $2 \mathrm{I} 2 \mathrm{O}$ configuration and using (11),

$$
\left\{\begin{array}{l}
z_{1} \\
z_{2} \\
z_{3}
\end{array}\right\}=\left[\begin{array}{ccc}
1 & 0 & 0 \\
0 & 1 & 0 \\
\sigma_{1} & \sigma_{2} & \sigma_{3}
\end{array}\right]\left\{\begin{array}{l}
x_{1} \\
x_{2} \\
x_{3}
\end{array}\right\}
$$

where

$$
\begin{gathered}
\sigma_{1}=\left(g_{21} g_{32}-g_{31} g_{22}\right), \quad \sigma_{2}=\left(g_{31} g_{12}-g_{11} g_{32}\right), \\
\sigma_{3}=\left(g_{11} g_{22}-g_{21} g_{12}\right)
\end{gathered}
$$

and $g_{i j}$ denotes the $i j$ th term of the matrix $\mathbf{G}$. Then, by inverting the transformation matrix $\mathbf{T}_{z x}$, the following expressions are obtained:

$$
x_{1}=z_{1}, \quad x_{2}=z_{2}, \quad x_{3}=-\frac{\sigma_{1}}{\sigma_{3}} z_{1}-\frac{\sigma_{2}}{\sigma_{3}} z_{2}+\frac{1}{\sigma_{3}} z_{3} .
$$

Then, from (15) and differentiating the bottom row of (B.1) it is found that

$$
\ddot{z}_{3}=\boldsymbol{\sigma}^{T} \mathbf{f}(\mathbf{z}, \dot{\mathbf{z}}), \quad \boldsymbol{\sigma}^{T}=\left\{\begin{array}{lll}
\sigma_{1} & \sigma_{2} & \sigma_{3}
\end{array}\right\},
$$

where

$$
\begin{aligned}
\mathbf{f}(\mathbf{z}, \dot{\mathbf{z}})= & \boldsymbol{\Phi}\left\{\dot{z}_{1} \quad \dot{z}_{2}\left(-\frac{\sigma_{1}}{\sigma_{3}} \dot{z}_{1}-\frac{\sigma_{2}}{\sigma_{3}} \dot{z}_{2}+\frac{1}{\sigma_{3}} \dot{z}_{3}\right)\right\}^{T} \\
& +\boldsymbol{\Psi}\left\{z_{1} \quad z_{2}\left(-\frac{\sigma_{1}}{\sigma_{3}} z_{1}-\frac{\sigma_{2}}{\sigma_{3}} z_{2}+\frac{1}{\sigma_{3}} z_{3}\right)\right\}^{T} \\
& +\boldsymbol{\Omega} \mathbf{f}_{\mathrm{nl}}(\mathbf{z}), \\
\mathbf{f}_{\mathrm{nl}}(\mathbf{z})= & K_{T}\left(-y_{2} z_{2}+\left(-\frac{\sigma_{1}}{\sigma_{3}} z_{1}-\frac{\sigma_{2}}{\sigma_{3}} z_{2}+\frac{1}{\sigma_{3}} z_{3}\right)\right)^{3} \\
& \times\left(\mathbf{T}_{(3,:)}\right)^{T} .
\end{aligned}
$$

Evidently, the internal dynamics expressions in (B.4) are nonlinear. Now, the zero dynamics may be obtained by setting to zero the coordinates corresponding to the external dynamics (i.e. the partially linearised system). In this case, the external coordinates are $z_{1}, z_{2}, \dot{z}_{1}, \dot{z}_{2}$ so that the zero dynamics are given by

$$
\begin{aligned}
\ddot{z}_{3, z d}= & \frac{1}{\sigma_{3}} \boldsymbol{\sigma}^{T} \boldsymbol{\Psi}_{(:, 3)} z_{3, z d}+\frac{1}{\sigma_{3}} \boldsymbol{\sigma}^{T} \boldsymbol{\Phi}_{(:, 3)} \dot{z}_{3, z d} \\
& +\frac{1}{\sigma_{3}^{3}} K_{T} \boldsymbol{\sigma}^{T} \boldsymbol{\Omega}\left(\mathbf{T}_{(3,:)}\right)^{T} z_{3, z d}^{3},
\end{aligned}
$$

where the subscript " $z d$ " signifies that the coordinates are specified under zero dynamics conditions.

Zero Dynamics Equilibrium Point Analysis. To obtain the equilibrium points of the zero dynamics, it is necessary to set $\ddot{z}_{3, z d}=\dot{z}_{3, z d}=0$. Equation (B.6) then produces

$$
\left(\boldsymbol{\sigma}^{T} \boldsymbol{\Psi}_{(:, 3)}+K_{T} \boldsymbol{\sigma}^{T} \boldsymbol{\Omega}\left(\mathbf{T}_{(3,:)}\right)^{T} z_{3, z d}^{2}\right) z_{3, z d}=0
$$

which provides the two solutions

$$
z_{3, z d}=0, \quad z_{3, z d}= \pm \sqrt{-\frac{\boldsymbol{\sigma}^{T} \Psi_{(:, 3)}}{K_{T} \boldsymbol{\sigma}^{T} \boldsymbol{\Omega}\left(\mathbf{T}_{(3,:)}\right)^{T}}} .
$$

It is found that the term inside the square root is always negative, and therefore the second solution for $z_{3, z d}$ is inadmissible. Thus, the only possible equilibrium point of the zero dynamics is the trivial solution

$$
\left\{\begin{array}{c}
z_{3} \\
\dot{z}_{3}
\end{array}\right\}_{z d}=\left\{\begin{array}{l}
0 \\
0
\end{array}\right\} \text {. }
$$


The nature of the above equilibrium point may be determined by examining the eigenvalues of (B.6), evaluated at the equilibrium point,

$$
\begin{aligned}
& s^{2}-\frac{1}{\sigma_{3}} \boldsymbol{\sigma}^{T} \boldsymbol{\Phi}_{(:, 3)} s-\frac{1}{\sigma_{3}} \boldsymbol{\sigma}^{T} \boldsymbol{\Psi}_{(:, 3)} \\
&-\frac{1}{\sigma_{3}^{3}} K_{T} \boldsymbol{\sigma}^{T} \boldsymbol{\Omega}\left(\mathbf{T}_{(3,:)}\right)^{T} z_{3, z d}^{2} \mid \begin{array}{l}
z_{3, z d}=0 \\
\dot{z}_{3, z d}=0
\end{array}=0,
\end{aligned}
$$

or

$$
s^{2}-\frac{1}{\sigma_{3}} \boldsymbol{\sigma}^{T} \boldsymbol{\Phi}_{(:, 3)} s-\frac{1}{\sigma_{3}} \boldsymbol{\sigma}^{T} \boldsymbol{\Psi}_{(:, 3)}=0 .
$$

The roots are found to be complex with negative real parts, thus revealing the type of equilibrium point to be a stable focus.

\section{Conflict of Interests}

The authors declare that there is no conflict of interests regarding the publication of this paper.

\section{Acknowledgment}

The authors wish to acknowledge the support of EPSRC Grant no. EP/J004987/1 on Nonlinear Active Vibration Suppression in Aeroelasticity.

\section{References}

[1] A. Isidori, Nonlinear Control Systems, Springer, New York, NY, USA, 1995.

[2] T. I. Fossen and M. J. Paulsen, "Adaptive feedback linearization applied to steering of ships," Modeling, Identification and Control, vol. 14, no. 4, pp. 229-237, 1993.

[3] J. Ko, A. J. Kurdila, and T. W. Strganac, "Nonlinear control of a prototypical wing section with torsional nonlinearity," Journal of Guidance, Control, and Dynamics, vol. 20, no. 6, pp. 1181-1189, 1997.

[4] J. Ko, A. J. Kurdila, and T. Strganac, "Stability and control of a structurally nonlinear aeroelastic system," Journal of Guidance, Control, and Dynamics, vol. 21, no. 5, pp. 718-725, 1998.

[5] J. Ko, T. W. Strganac, and A. J. Kurdila, "Adaptive feedback linearization for the control of a typical wing section with structural nonlinearity," Nonlinear Dynamics, vol. 18, no. 3, pp. 289-301, 1999.

[6] M. M. Monahemi and M. Krstic, "Control of wing rock motion using adaptive feedback linearization," Journal of Guidance, Control, and Dynamics, vol. 19, no. 4, pp. 905-912, 1996.

[7] A. Poursamad, "Adaptive feedback linearization control of antilock braking systems using neural networks," Mechatronics, vol. 19, no. 5, pp. 767-773, 2009.

[8] C. P. Bechlioulis and G. A. Rovithakis, "Robust adaptive control of feedback linearizable MIMO nonlinear systems with prescribed performance," IEEE Transactions on Automatic Control, vol. 53, no. 9, pp. 2090-2099, 2008.

[9] K. Shojaei, A. Mohammad Shahri, and A. Tarakameh, "Adaptive feedback linearizing control of nonholonomic wheeled mobile robots in presence of parametric and nonparametric uncertainties," Robotics and Computer-Integrated Manufacturing, vol. 27, no. 1, pp. 194-204, 2011.

[10] L. Tuan, S.-G. Lee, V.-H. Dang, S. Moon, and B. Kim, "Partial feedback linearization control of a three-dimensional overhead crane," International Journal of Control, Automation and Systems, vol. 11, no. 4, pp. 718-727, 2013.

[11] Y. M. Ram and J. E. Mottershead, "Multiple-input active vibration control by partial pole placement using the method of receptances," Mechanical Systems and Signal Processing, vol. 40, no. 2, pp. 727-735, 2013.

[12] J. R. Wright and J. E. Cooper, Introduction to Aircraft Aeroelasticity and Loads, Wiley, Chichester, UK, 2007.

[13] S. Jiffri, J. E. Mottershead, and J. E. Cooper, "Adaptive feedback linearisation and control of a flexible aircraft wing," in Topics in Modal Analysis, Volume 7, Conference Proceedings of the Society for Experimental Mechanics Series, Springer, 2013.

[14] http://www.airbus.com/aircraftfamilies/passengeraircraft/ a330family/a330-200/specifications/.

[15] D. P. Atherton, Stability of Nonlinear Systems, John Wiley \& Sons, New York, NY, USA, 1981. 

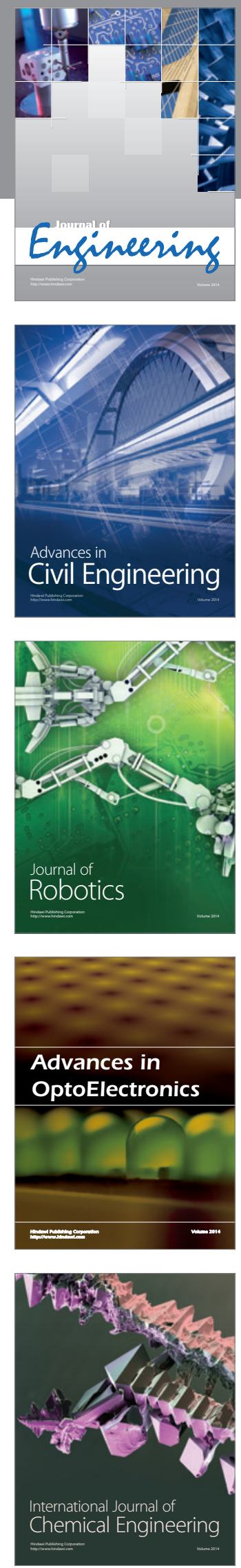

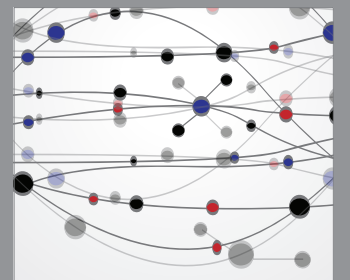

The Scientific World Journal
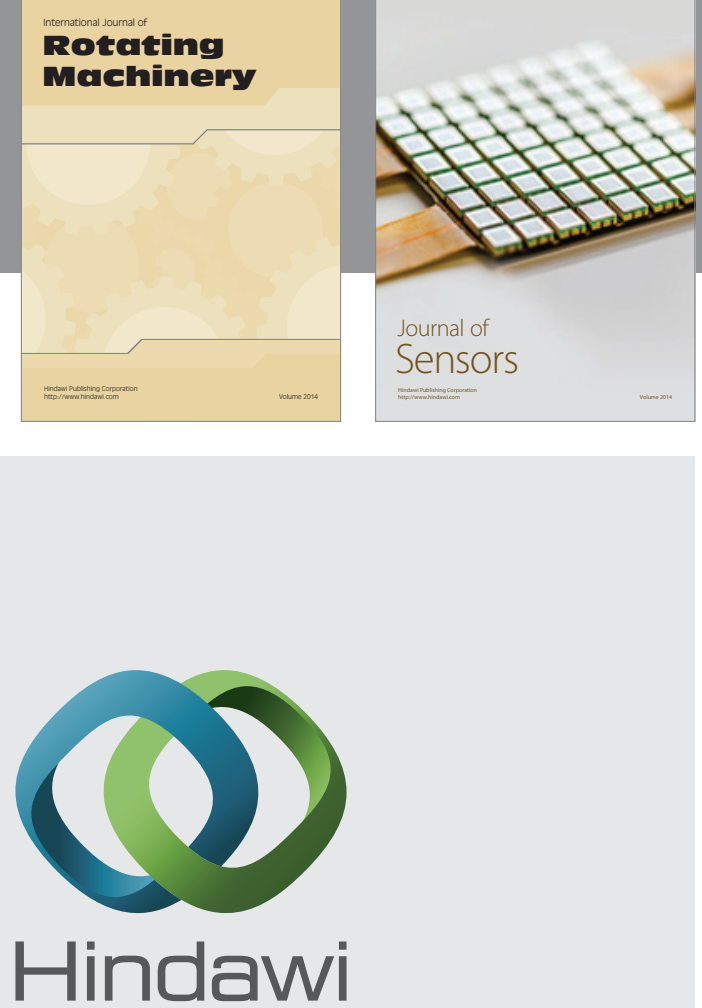

Submit your manuscripts at http://www.hindawi.com
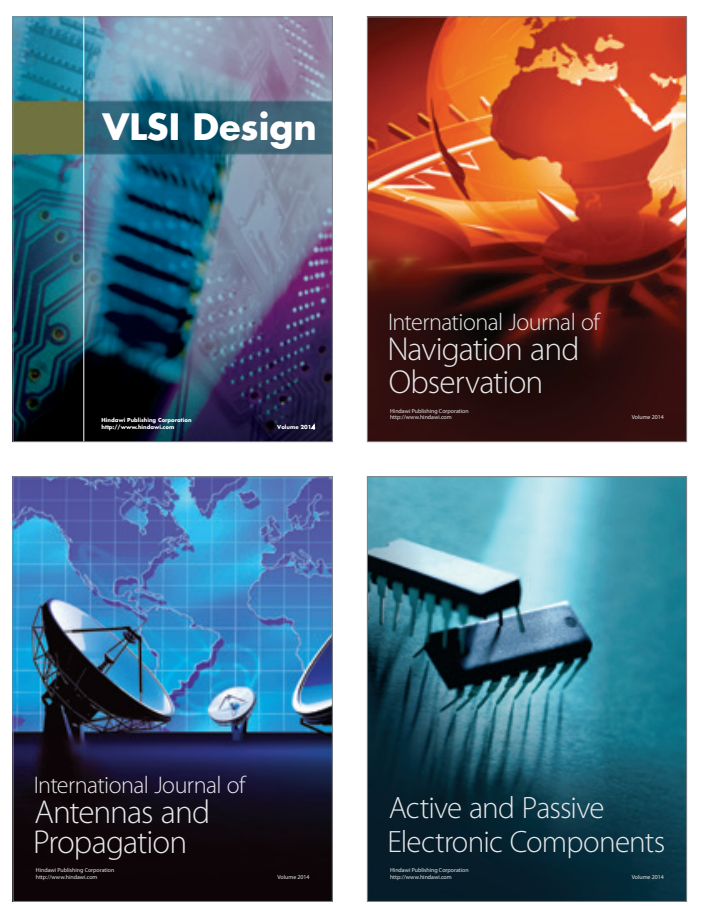
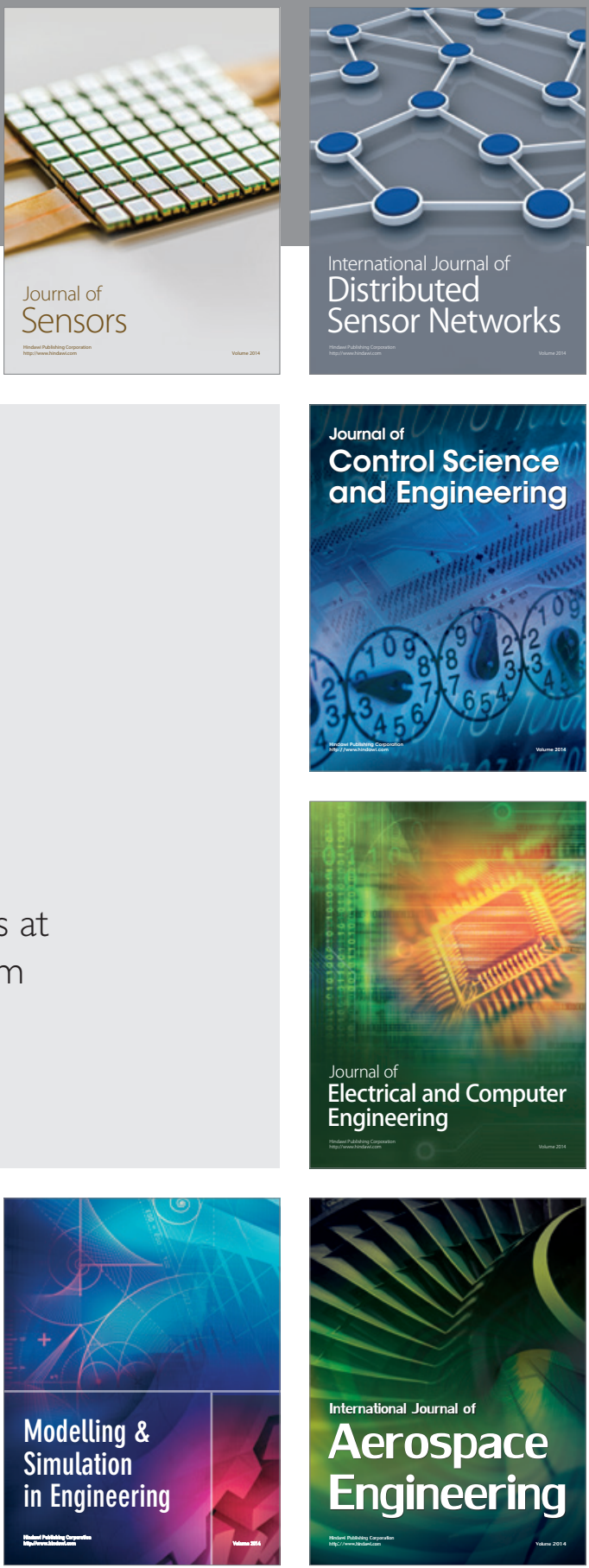

Journal of

Control Science

and Engineering
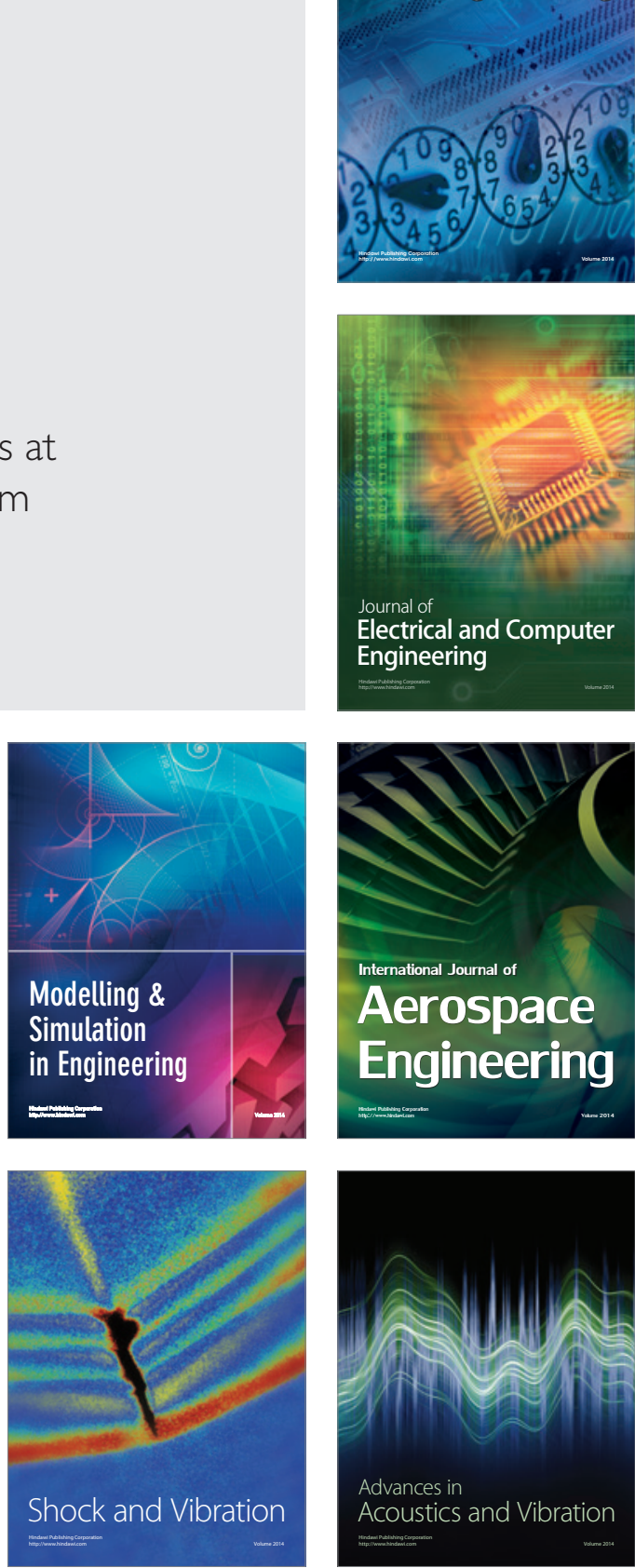